\title{
A concave-convex elliptic problem involving the fractional Laplacian
}

\author{
C. Brändle, E. Colorado, A. de Pablo and U. Sánchez \\ Departamento de Matemáticas, Universidad Carlos III de Madrid, \\ 28911 Leganés, Madrid, Spain (eduardo.colorado@uc3m.es)
}

(MS received 25 January 2011; accepted 2 November 2011)

We study a nonlinear elliptic problem defined in a bounded domain involving fractional powers of the Laplacian operator together with a concave-convex term. We completely characterize the range of parameters for which solutions of the problem exist and prove a multiplicity result. We also prove an associated trace inequality and some Liouville-type results.

\section{Introduction}

Over the last few decades the problem

$$
\begin{array}{clrl}
-\Delta u=f(u) & & \text { in } \Omega \subset \mathbb{R}^{N}, \\
u=0 & & \text { on } \partial \Omega,
\end{array}
$$

has been widely investigated (for a survey see [3], and for more specific problems see, for example, $[5,13,36]$, where different nonlinearities and different classes of domains, bounded and unbounded, are considered). Other different diffusion operators, like the $p$-Laplacian, fully nonlinear operators, etc., have also been studied (see, for example, $[9,16,27]$ and the references therein). We deal with a non-local version of the above problem for a particular type of nonlinearities, i.e. we study a concave-convex problem involving the fractional Laplacian operator

$$
\left.\begin{array}{c}
(-\Delta)^{\alpha / 2} u=\lambda u^{q}+u^{p}, \quad u>0 \text { in } \Omega, \\
u=0 \quad \text { on } \partial \Omega,
\end{array}\right\}
$$

with $0<\alpha<2,0<q<1<p<(N+\alpha) /(N-\alpha), N>\alpha, \lambda>0$ and where $\Omega \subset \mathbb{R}^{N}$ is a smooth bounded domain. The critical case $p=(N+\alpha) /(N-\alpha)$ is studied in [7].

The non-local operator $(-\Delta)^{\alpha / 2}$ in $\mathbb{R}^{N}$ is defined on the Schwartz class of functions $g \in \mathcal{S}$ through the Fourier transform

$$
\left[(-\Delta)^{\alpha / 2} g\right]^{\wedge}(\xi)=(2 \pi|\xi|)^{\alpha} \hat{g}(\xi),
$$

or via the Riesz potential (see, for example, $[33,40]$ ). Observe that $\alpha=2$ corresponds to the standard local Laplacian.

This type of diffusion operators arises in several areas such as physics, probability and finance (see, for example, $[6,8,23,44]$ ). In particular, the fractional Laplacian can be understood as the infinitesimal generator of a stable Lévy process [8].

(C) 2013 The Royal Society of Edinburgh 
There is another way of defining this operator. In fact, in the case $\alpha=1$, there is an explicit means of calculating the half-Laplacian acting on a function $u$ in the whole space $\mathbb{R}^{N}$ as the normal derivative on the boundary of its harmonic extension to the upper half-space $\mathbb{R}_{+}^{N+1}$ : the so-called Dirichlet-to-Neumann operator. The ' $\alpha$ derivative' $(-\Delta)^{\alpha / 2}$ can be characterized in a similar way, defining the $\alpha$-harmonic extension to the upper half-space (see [17] and $\S 2$ for details). This extension is commonly used in the recent literature since it allows non-local problems to be written in a local way and this permits the use of variational techniques for these kind of problems.

In cases where the operator is defined in bounded domains $\Omega$, the above characterization has to be adapted. The fractional powers of a linear positive operator in $\Omega$ are defined by means of the spectral decomposition. In [15], the fractional operator $(-\Delta)^{1 / 2}$ is considered, defined using the mentioned Dirichlet-to-Neumann operator, but restricted to the cylinder $\Omega \times \mathbb{R}_{+} \subset \mathbb{R}_{+}^{N+1}$, and it is shown that this definition is coherent with the spectral one (see also [41] for the case where $\alpha \neq 1$ ). We recall that this is not the unique possibility of defining a non-local operator related to the fractional Laplacian in a bounded domain (see, for example, the definition of the so-called regional fractional Laplacian in [10,31], where the authors consider the Riesz integral restricted to the domain $\Omega$ ). This leads to a different operator related to a Neumann problem.

There are many results on the subject of concave-convex nonlinearity involving different (local) operators (see, for example, $[1,5,9,18,22,27]$ ). Some ideas in the present paper are taken from [5]. In most of the problems considered in the aforementioned works, a critical exponent appears, which separates generically the ranges where compactness results can or cannot be applied (in the fully nonlinear case, the situation is slightly different, but a critical exponent still appears [18]). In our case, the critical exponent with respect to the corresponding Sobolev embedding is given by $2_{\alpha}^{*}=2 N /(N-\alpha)$. This is a reason why problem (1.1) is studied in the subcritical case $p<2_{\alpha}^{*}-1=(N+\alpha) /(N-\alpha)$ (see also the non-existence result for supercritical nonlinearities in corollary 5.6).

The main results proven here characterize the existence of solutions of (1.1) in terms of the parameter $\lambda$. The competition between the sublinear and superlinear terms plays a role, which leads to different results concerning the existence and multiplicity of solutions, among other things. By 'solution' we mean an energy solution (see the precise definition in $\S 5$ ).

THEOREM 1.1. There exists $\Lambda>0$ such that, for problem (1.1), the following hold.

(i) If $0<\lambda<\Lambda$, there is a minimal solution. Moreover, the family of minimal solutions is increasing with respect to $\lambda$.

(ii) If $\lambda=\Lambda$, there is at least one solution.

(iii) If $\lambda>\Lambda$, there is no solution.

(iv) For any $0<\lambda<\Lambda$, there exist at least two solutions.

For $\alpha \in[1,2)$ and $p$ subcritical, we also prove that there exists a universal $L^{\infty}$ bound for every solution to problem (1.1) independently of $\lambda$. 
Theorem 1.2. Let $\alpha \geqslant 1$. Then there exists a constant $C>0$ such that, for any $0 \leqslant \lambda \leqslant \Lambda$, every solution to problem (1.1) satisfies

$$
\|u\|_{\infty} \leqslant C
$$

The proof of this last result relies on the classical argument of rescaling introduced in [28], which yields to problems on unbounded domains. Therefore, some Liouvilletype results are required, and this is the point where the restriction $\alpha \geqslant 1$ appears.

The paper is organized as follows. We devote $\S \S 2$ and 3 to studying the fractional Laplacian in the whole space by means of the $\alpha$-harmonic extension and obtain a trace inequality and two non-existence results. In $\S 4$ we consider the $\alpha$-harmonic extension in cylinders in order to define the fractional Laplacian in a bounded domain. We also study an associated linear equation in the local version. Finally, $\S 5$ contains the results related to the non-local nonlinear problem (1.1), where we prove theorems 1.1 and 1.2.

\section{The fractional Laplacian in the whole space}

In order to study problem (1.1), we need to properly define the fractional Laplacian in bounded domains. This is done in $\S 4$. Prior to this, we recall the definition of the fractional Laplacian in the whole space and some of its properties. More specifically, we obtain a trace inequality that is relevant in what follows and which also has interest in itself.

\subsection{Preliminaries}

The fractional Laplacian is defined in the whole space through the Fourier transform (see (1.2)). Recently, it was shown in [17] that this operator can be realized in a local way by using one more variable and the so-called $\alpha$-harmonic extension. This is a main tool in our investigation.

More precisely, if $u$ is a regular function in $\mathbb{R}^{N}$, we say that $w=E_{\alpha}(u)$ is its $\alpha$ harmonic extension to the upper half-space, $\mathbb{R}_{+}^{N+1}$, if $w$ is a solution to the problem

$$
\begin{aligned}
-\operatorname{div}\left(y^{1-\alpha} \nabla w\right)=0 & & \text { in } \mathbb{R}_{+}^{N+1}, \\
w=u & & \text { on } \mathbb{R}^{N} \times\{y=0\} .
\end{aligned}
$$

In $[17]$ it is proved that

$$
\lim _{y \rightarrow 0^{+}} y^{1-\alpha} \frac{\partial w}{\partial y}(x, y)=-\kappa_{\alpha}(-\Delta)^{\alpha / 2} u(x),
$$

where

$$
\kappa_{\alpha}=\frac{2^{1-\alpha} \Gamma\left(1-\frac{1}{2} \alpha\right)}{\Gamma\left(\frac{1}{2} \alpha\right)}
$$

The appropriate functional spaces to work with are

$$
X^{\alpha}\left(\mathbb{R}_{+}^{N+1}\right) \text { and } \dot{H}^{\alpha / 2}\left(\mathbb{R}^{N}\right),
$$


defined as the completion of $\mathcal{C}_{0}^{\infty}\left(\overline{\mathbb{R}_{+}^{N+1}}\right)$ and $\mathcal{C}_{0}^{\infty}\left(\mathbb{R}^{N}\right)$, respectively, under the norms

$$
\begin{aligned}
\|\phi\|_{X^{\alpha}}^{2} & =\int_{\mathbb{R}_{+}^{N+1}} y^{1-\alpha}|\nabla \phi(x, y)|^{2} \mathrm{~d} x \mathrm{~d} y, \\
\|\psi\|_{\dot{H}^{\alpha / 2}}^{2} & =\int_{\mathbb{R}^{N}}|2 \pi \xi|^{\alpha}|\hat{\psi}(\xi)|^{2} \mathrm{~d} \xi \\
& =\int_{\mathbb{R}^{N}}\left|(-\Delta)^{\alpha / 4} \psi(x)\right|^{2} \mathrm{~d} x .
\end{aligned}
$$

The extension operator is well defined for smooth functions through a Poisson kernel, whose explicit expression is given in [17]. It can also be defined in the space $\dot{H}^{\alpha / 2}\left(\mathbb{R}^{N}\right)$, and, in fact,

$$
\left\|E_{\alpha}(\psi)\right\|_{X^{\alpha}}=c_{\alpha}\|\psi\|_{\dot{H}^{\alpha / 2}} \quad \text { for all } \psi \in \dot{H}^{\alpha / 2}\left(\mathbb{R}^{N}\right),
$$

where $c_{\alpha}=\sqrt{\kappa_{\alpha}}$ (see lemma 2.2). On the other hand, for a function $\phi \in X^{\alpha}\left(\mathbb{R}_{+}^{N+1}\right)$, we shall denote its trace on $\mathbb{R}^{N} \times\{y=0\}$ as $\operatorname{tr}(\phi)$. This trace operator is also well defined and it satisfies

$$
\|\operatorname{tr}(\phi)\|_{\dot{H}^{\alpha / 2}} \leqslant c_{\alpha}^{-1}\|\phi\|_{X^{\alpha}}
$$

\subsection{A trace inequality}

From expression (2.3), the Sobolev embedding implies that the trace also belongs to $L^{2_{\alpha}^{*}}\left(\mathbb{R}^{N}\right)$, where $2_{\alpha}^{*}=2 N /(N-\alpha)$. Even the best constant associated to this inclusion is attained and can be characterized. Although most of the results used in order to prove the following theorem are known, we have collected them here for the reader's convenience.

THEOREM 2.1. For every $z \in X^{\alpha}\left(\mathbb{R}_{+}^{N+1}\right)$, it holds that

$$
\left(\int_{\mathbb{R}^{N}}|v(x)|^{2 N /(N-\alpha)} \mathrm{d} x\right)^{(N-\alpha) / N} \leqslant S(\alpha, N) \int_{\mathbb{R}_{+}^{N+1}} y^{1-\alpha}|\nabla z(x, y)|^{2} \mathrm{~d} x \mathrm{~d} y,
$$

where $v=\operatorname{tr}(z)$. The best constant takes the exact value

$$
S(\alpha, N)=\frac{\Gamma\left(\frac{1}{2} \alpha\right) \Gamma\left(\frac{1}{2}(N-\alpha)\right)(\Gamma(N))^{\alpha / N}}{2 \pi^{\alpha / 2} \Gamma\left(\frac{1}{2}(2-\alpha)\right) \Gamma\left(\frac{1}{2}(N+\alpha)\right)\left(\Gamma\left(\frac{1}{2} N\right)\right)^{\alpha / N}}
$$

and it is achieved when $v$ takes the form

$$
v(x)=\tau^{(N-\alpha) / 2}\left(\left|x-x_{0}\right|^{2}+\tau^{2}\right)^{-(N-\alpha) / 2}
$$

for some $x_{0} \in \mathbb{R}^{N}, \tau>0$ and $z=E_{\alpha}(v)$.

The analogous results for the classical Laplace operator can be found in $[25,36]$.

Lemma 2.2. Let $v \in \dot{H}^{\alpha / 2}\left(\mathbb{R}^{N}\right)$, and let $z=E_{\beta}(v)$ be its $\beta$-harmonic extension, $\beta \in\left(\frac{1}{2} \alpha, 2\right)$. Then $z \in X^{\alpha}\left(\mathbb{R}_{+}^{N+1}\right)$ and, moreover, there exists a positive universal constant $c(\alpha, \beta)$ such that

$$
\|v\|_{\dot{H}^{\alpha / 2}}=c(\alpha, \beta)\|z\|_{X^{\alpha}} .
$$

In particular, if $\beta=\alpha$, we have $c(\alpha, \alpha)=1 / \sqrt{\kappa_{\alpha}}$. 
Inequality (2.4) only needs the case $\beta=\alpha$, which is deduced directly from the proof of the local characterization of $(-\Delta)^{\alpha / 2}$ in [17]. The calculations performed in [17] can be extended to cover the range $\frac{1}{2} \alpha<\beta<2$, including, in particular, the case $\beta=1$ proved in [45].

Proof. Since $z=E_{\beta}(v)$, by definition $z$ solves $\operatorname{div}\left(y^{1-\beta} \nabla z\right)=0$, which is equivalent to

$$
\Delta_{x} z+\frac{1-\beta}{y} \frac{\partial z}{\partial y}+\frac{\partial^{2} z}{\partial y^{2}}=0
$$

Taking the Fourier transform in $x \in \mathbb{R}^{N}$ for fixed $y>0$, we have

$$
-4 \pi^{2}|\xi|^{2} \hat{z}+\frac{1-\beta}{y} \frac{\partial \hat{z}}{\partial y}+\frac{\partial^{2} \hat{z}}{\partial y^{2}}=0
$$

and $\hat{z}(\xi, 0)=\hat{v}(\xi)$. Therefore, $\hat{z}(\xi, y)=\hat{v}(\xi) \phi_{\beta}(2 \pi|\xi| y)$, where $\phi_{\beta}$ solves the problem

$$
-\phi+\frac{1-\beta}{s} \phi^{\prime}+\phi^{\prime \prime}=0, \quad \phi(0)=1, \quad \lim _{s \rightarrow \infty} \phi(s)=0 .
$$

In fact, $\phi_{\beta}$ minimizes the functional

$$
H_{\beta}(\phi)=\int_{0}^{\infty}\left(|\phi(s)|^{2}+\left|\phi^{\prime}(s)\right|^{2}\right) s^{1-\beta} \mathrm{d} s
$$

and it can be shown that it is a combination of Bessel functions [34]. More precisely, $\phi_{\beta}$ satisfies the following asymptotic behaviour:

$$
\phi_{\beta}(s) \sim \begin{cases}1-c_{1} s^{\beta} & \text { for } s \rightarrow 0, \\ c_{2} s^{(\beta-1) / 2} \mathrm{e}^{-s} & \text { for } s \rightarrow \infty,\end{cases}
$$

where

$$
c_{1}(\beta)=\frac{2^{1-\beta} \Gamma\left(1-\frac{1}{2} \beta\right)}{\beta \Gamma\left(\frac{1}{2} \beta\right)}, \quad c_{2}(\beta)=\frac{2^{(1-\beta) / 2} \pi^{1 / 2}}{\Gamma\left(\frac{1}{2} \beta\right)} .
$$

Now we observe that

$$
\begin{aligned}
\int_{\mathbb{R}^{N}}|\nabla z(x, y)|^{2} \mathrm{~d} x & =\int_{\mathbb{R}^{N}}\left(\left|\nabla_{x} z(x, y)\right|^{2}+\left|\frac{\partial z}{\partial y}(x, y)\right|^{2}\right) \mathrm{d} x \\
& =\int_{\mathbb{R}^{N}}\left(4 \pi^{2}|\xi|^{2}|\hat{z}(\xi, y)|^{2}+\left|\frac{\partial \hat{z}}{\partial y}(\xi, y)\right|^{2}\right) \mathrm{d} \xi
\end{aligned}
$$

Then, multiplying by $y^{1-\alpha}$ and integrating in $y$,

$$
\begin{aligned}
\int_{0}^{\infty} \int_{\mathbb{R}^{N}} & y^{1-\alpha}|\nabla z(x, y)|^{2} \mathrm{~d} x \mathrm{~d} y \\
& =\int_{0}^{\infty} \int_{\mathbb{R}^{N}} 4 \pi^{2}|\xi|^{2}|\hat{v}(\xi)|^{2}\left(\left|\phi_{\beta}(2 \pi|\xi| y)\right|^{2}+\left|\phi_{\beta}^{\prime}(2 \pi|\xi| y)\right|^{2}\right) y^{1-\alpha} \mathrm{d} \xi \mathrm{d} y \\
& =\int_{0}^{\infty}\left(\left|\phi_{\beta}(s)\right|^{2}+\left|\phi_{\beta}^{\prime}(s)\right|^{2}\right) s^{1-\alpha} \mathrm{d} s \int_{\mathbb{R}^{N}}|2 \pi \xi|^{\alpha}|\hat{v}(\xi)|^{2} \mathrm{~d} \xi
\end{aligned}
$$


Using (2.9), we see that the integral

$$
\int_{0}^{\infty}\left(\left|\phi_{\beta}\right|^{2}+\left|\phi_{\beta}^{\prime}\right|^{2}\right) s^{1-\alpha} \mathrm{d} s
$$

is convergent provided $\beta>\frac{1}{2} \alpha$. This proves (2.7) with $c(\alpha, \beta)=\left(H_{\alpha}\left(\phi_{\beta}\right)\right)^{-1 / 2}$.

REMARK 2.3. If $\beta=1$, we have $\phi_{1}(s)=\mathrm{e}^{-s}$, and $H_{\alpha}\left(\phi_{1}\right)=2^{\alpha-1} \Gamma(2-\alpha)$ [45]. Moreover, when $\beta=\alpha$, integrating by parts and using the equation in (2.8), with (2.9), we obtain

$$
\begin{aligned}
H_{\alpha}\left(\phi_{\alpha}\right) & =\int_{0}^{\infty}\left[\phi_{\alpha}^{2}(s)+\left(\phi_{\alpha}^{\prime}\right)^{2}(s)\right] s^{1-\alpha} \mathrm{d} s \\
& =-\lim _{s \rightarrow 0} s^{1-\alpha} \phi_{\alpha}^{\prime}(s) \\
& =\alpha c_{1}(\alpha) \\
& =\kappa_{\alpha} .
\end{aligned}
$$

LEMMA 2.4. Let $z \in X^{\alpha}\left(\mathbb{R}_{+}^{N+1}\right)$ and let $w=E_{\alpha}(\operatorname{tr}(z))$ be its $\alpha$-harmonic associated function (the extension of the trace). Then

$$
\|z\|_{X^{\alpha}}^{2}=\|w\|_{X^{\alpha}}^{2}+\|z-w\|_{X^{\alpha}}^{2} .
$$

Proof. Observe that, for $h=z-w$, we have

$$
\|z\|_{X^{\alpha}}^{2}=\int_{\mathbb{R}_{+}^{N+1}} y^{1-\alpha}\left(|\nabla w|^{2}+|\nabla h|^{2}+2\langle\nabla w, \nabla h\rangle\right) .
$$

But, since $\operatorname{tr}(h)=0$, we have

$$
\int_{\mathbb{R}_{+}^{N+1}} y^{1-\alpha}\langle\nabla w, \nabla h\rangle \mathrm{d} x \mathrm{~d} y=0 .
$$

Lemma 2.5. If $g \in L^{2 N /(N+\alpha)}\left(\mathbb{R}^{N}\right)$, and $f \in \dot{H}^{\alpha / 2}\left(\mathbb{R}^{N}\right)$, then there exists a constant $\ell(\alpha, N)>0$ such that

$$
\left|\int f(x) g(x) \mathrm{d} x\right| \leqslant \ell(\alpha, N)\|f\|_{\dot{H}^{\alpha / 2}}\|g\|_{2 N /(N+\alpha)} .
$$

Moreover, the equality in (2.11) with the best constant holds when $f$ and $g$ take the form (2.6). 45].

The proof follows by a standard argument that can be found, for example, in [24,

Proof. By Parçeval's identity and the Cauchy-Schwarz inequality, we have

$$
\begin{aligned}
\left(\int_{\mathbb{R}^{N}} f(x) g(x) \mathrm{d} x\right)^{2} & =\left(\int_{\mathbb{R}^{N}} \hat{f}(\xi) \hat{g}(\xi) \mathrm{d} \xi\right)^{2} \\
& \leqslant\left(\int_{\mathbb{R}^{N}}|2 \pi \xi|^{\alpha}|\hat{f}(\xi)|^{2} \mathrm{~d} \xi\right)\left(\int_{\mathbb{R}^{N}}|2 \pi \xi|^{-\alpha}|\hat{g}(\xi)|^{2} \mathrm{~d} \xi\right) .
\end{aligned}
$$


The second term can be written using the results of [35] as

$$
\int_{\mathbb{R}^{N}}|2 \pi \xi|^{-\alpha}|\hat{g}(\xi)|^{2} \mathrm{~d} \xi=b(\alpha, N) \int_{\mathbb{R}^{2 N}} \frac{g(x) g\left(x^{\prime}\right)}{\left|x-x^{\prime}\right|^{N-\alpha}} \mathrm{d} x \mathrm{~d} x^{\prime}
$$

where

$$
b(\alpha, N)=\frac{\Gamma\left(\frac{1}{2}(N-\alpha)\right)}{2^{\alpha} \pi^{N / 2} \Gamma\left(\frac{1}{2} \alpha\right)} .
$$

We now use the following Hardy-Littlewood-Sobolev inequality [35],

$$
\int_{\mathbb{R}^{2 N}} \frac{g(x) g\left(x^{\prime}\right)}{\left|x-x^{\prime}\right|^{N-\alpha}} \mathrm{d} x \mathrm{~d} x^{\prime} \leqslant d(\alpha, N)\|g\|_{2 N /(N+\alpha)}^{2},
$$

where

$$
d(\alpha, N)=\frac{\pi^{(N-\alpha) / 2} \Gamma\left(\frac{1}{2} \alpha\right)(\Gamma(N))^{\alpha / N}}{\Gamma\left(\frac{1}{2}(N+\alpha)\right)\left(\Gamma\left(\frac{1}{2} N\right)\right)^{\alpha / N}},
$$

with equality if $g$ takes the form (2.6). From this, we obtain the desired estimate $(2.11)$ with the constant $\ell(\alpha, N)=\sqrt{b(\alpha, N) d(\alpha, N)}$.

When applying the Cauchy-Schwarz inequality, we obtain an identity provided the functions $|\xi|^{\alpha / 2} \hat{f}(\xi)$ and $|\xi|^{-\alpha / 2} \hat{g}(\xi)$ are proportional. This means

$$
\hat{g}(\xi)=c|\xi|^{\alpha} \hat{f}(\xi)=c\left[(-\Delta)^{\alpha / 2} f\right]^{\wedge}(\xi) .
$$

We conclude by observing that if $g$ takes the form (2.6) and $g=c(-\Delta)^{\alpha / 2} f$, then $f$ also takes the form (2.6).

Proof of theorem 2.1. We apply lemma 2.5 with $g=|f|^{(N+\alpha) /(N-\alpha)-1} f$, then use lemma 2.2 and conclude using lemma 2.4. The best constant is

$$
S(\alpha, N)=\frac{\ell^{2}(\alpha, N)}{\kappa_{\alpha}} .
$$

Related to this result, we cite [20], where it is proved that the only positive regular solutions to $(-\Delta)^{\alpha / 2} f=c f^{(N+\alpha) /(N-\alpha)}$ take the form (2.6).

Remark 2.6. If we let $\alpha$ tend to 2 , when $N>2$, we recover the classical Sobolev inequality for a function in $H^{1}\left(\mathbb{R}^{N}\right)$, with the same constant [43]. In order to pass to the limit on the right-hand side of (2.4), at least formally, we observe that $(2-\alpha) y^{1-\alpha} \mathrm{d} y$ is a measure on compact sets of $\mathbb{R}_{+}$converging (in the weak* sense) to a Dirac delta. Hence,

$$
\lim _{\alpha \rightarrow 2^{-}} \int_{0}^{1}\left(\int_{\mathbb{R}^{N}}|\nabla z(x, y)|^{2} \mathrm{~d} x\right)(2-\alpha) y^{1-\alpha} \mathrm{d} y=\int_{\mathbb{R}^{N}}|\nabla v(x)|^{2} \mathrm{~d} x .
$$

We then obtain

$$
\left(\int_{\mathbb{R}^{N}}|v(x)|^{2 N /(N-2)} \mathrm{d} x\right)^{(N-2) / N} \leqslant S(N) \int_{\mathbb{R}^{N}}|\nabla v(x)|^{2} \mathrm{~d} x,
$$


with the best constant

$$
\begin{aligned}
S(N) & =\lim _{\alpha \rightarrow 2^{-}} \frac{S(\alpha, N)}{2-\alpha} \\
& =\frac{1}{\pi N(N-2)}\left(\frac{\Gamma(N)}{\Gamma\left(\frac{1}{2} N\right)}\right)^{2 / N} .
\end{aligned}
$$

It is achieved when $v$ takes the form (2.6) with $\alpha$ replaced by 2 .

\section{Some non-existence results in unbounded domains}

In this section we prove two Liouville-type results in the half-space $\mathbb{R}_{+}^{N+1}$ and the quarter-space $\mathbb{R}_{++}^{N+1}$ that will be useful in $\S 5.4$ in order to obtain uniform a priori bounds for the solutions to problem (1.1). These results have a corresponding formulation for the fractional Laplacian operator.

\subsection{A problem in the half-space}

THEOREM 3.1. Let $1 \leqslant \alpha<2$. Then the problem in the half-space $\mathbb{R}_{+}^{N+1}$,

$$
\left.\begin{array}{rlrl}
-\operatorname{div}\left(y^{1-\alpha} \nabla w\right) & =0 & & \text { in } \mathbb{R}_{+}^{N+1}, \\
\frac{\partial w}{\partial \nu^{\alpha}}(x) & =w^{p}(x, 0) & & \text { on } \partial \mathbb{R}_{+}^{N+1}=\mathbb{R}^{N},
\end{array}\right\}
$$

has no positive bounded solution provided $p<(N+\alpha) /(N-\alpha)$.

Theorem 3.1 is proved in the case $\alpha=1$ in [32]. On the other hand, Chen et al. [19] consider the corresponding non-local problem and perform a different proof of the same result, using integral estimates, which is valid in the whole interval $0<\alpha<2$.

The proof that we present here is based on the well-known method of moving planes, introduced by Alexandrov and first used in the context of partial differential equations by $[29,39]$, among others.

We begin by establishing some useful notation in order to apply the moving planes method. The points of the upper half-space $\mathbb{R}_{+}^{N+1}$ are denoted by $X=(x, y)$, where $x=\left(x_{1}, \ldots, x_{N}\right)$ and $y>0$. Fix $\rho>0$ and consider the sets

$$
\Sigma_{\rho}=\left\{X \in \mathbb{R}_{+}^{N+1} ; x_{N}>\rho\right\}, \quad T_{\rho}=\left\{X \in \overline{\mathbb{R}_{+}^{N+1}} ; x_{N}=\rho\right\} .
$$

For every $X=(x, y) \in \mathbb{R}_{+}^{N+1}$ we define the reflection across the hyperplane $T_{\rho}$ by

$$
X^{\rho}=\left(x^{\rho}, y\right)=X+2\left(\rho-x_{N}\right) e_{N}=\left(x_{1}, \ldots, 2 \rho-x_{N}, y\right) .
$$

Let us also consider the point $P_{\rho}=(0, \ldots, 0,2 \rho, 0) \in \Sigma_{\rho}$, whose reflection is the origin, and the set $\tilde{\Sigma}_{\rho}=\bar{\Sigma}_{\rho} \backslash\left\{P_{\rho}\right\}$. Let $B_{r}^{+}$denote the half-ball $B_{r}^{+}=\{|X|<$ $r, y>0\}\left(B_{r}^{+}\left(X_{0}\right)\right.$ when the centre $X_{0}=\left(x_{0}, 0\right)$ is not the origin), and let its non-flat part of the boundary be denoted by $S_{r}^{+}=\{|X|=r, y>0\}$ (respectively, $\left.S_{r}^{+}\left(X_{0}\right)\right)$.

We also define the operator

$$
-L_{\alpha} w=y^{\alpha-1} \operatorname{div}\left(y^{1-\alpha} \nabla w\right)=\Delta w+\frac{1-\alpha}{y} \frac{\partial w}{\partial y}
$$


in such a way that problem (3.1) can be written in the form

$$
\left.\begin{array}{ll}
L_{\alpha} w=0 & \text { in } \mathbb{R}_{+}^{N+1}, \\
\frac{\partial w}{\partial \nu^{\alpha}}=w^{p} & \text { for } y=0 .
\end{array}\right\}
$$

Two easy properties of the operator $L_{\alpha}$ are used in the following:

$$
\left.\begin{array}{rl}
L_{\alpha}(f g) & =f L_{\alpha} g+g L_{\alpha} f+2\langle\nabla f, \nabla g\rangle, \\
L_{\alpha}\left(|X|^{\gamma}\right) & =\gamma(\gamma+N-\alpha)|X|^{\gamma-2} .
\end{array}\right\}
$$

Finally, the so-called fractional Kelvin transform will also be useful. We consider, for a function $f$ defined in $\mathbb{R}^{N}$, its fractional Kelvin transform as $K_{\alpha}(f)(x)=$ $|x|^{\alpha-N} f\left(x /|x|^{2}\right)$. It is well known that this transform behaves under the action of the fractional Laplacian in a similar way to the standard Kelvin transform with the Laplacian

$$
(-\Delta)^{\alpha / 2} K_{\alpha}(f)(x)=|x|^{-\alpha-N}(-\Delta)^{\alpha / 2} f\left(x /|x|^{2}\right) .
$$

We are interested in defining the analogous fractional Kelvin transform for the function $w$ and the operator $L_{\alpha}$. Let $z(X)=|X|^{\gamma} w(\xi), \xi=X /|X|^{2}$. It is a matter of calculus to see that

$$
L_{\alpha} z(X)=|X|^{\gamma-4}\left(L_{\alpha} w(\xi)+(\gamma+N-\alpha)|X|^{2}(\gamma w(\xi)-2\langle\xi, \nabla w(\xi)\rangle)\right) .
$$

Therefore, if we choose $\gamma=\alpha-N$ and $w$ is $\alpha$-harmonic, we obtain that $z$ is also $\alpha$-harmonic, and so it turns out to be the $\alpha$-harmonic extension of $K_{\alpha}(f)$ if $w$ is the $\alpha$-harmonic extension of $f$. In other words, $E \circ K_{\alpha}=K_{\alpha} \circ E$.

Now let $w$ be any solution to problem (3.4), and set $\mu=\sup _{B_{1}^{+}} w$. Then there exists $\varepsilon>0$ such that $w(X) \geqslant \varepsilon|X|^{\alpha-N}$ for $|X| \geqslant 1, y>0$. To see this, observe that, by the Harnack inequality [14, lemma 4.8], we have $\varepsilon=\inf _{S_{1}^{+}} w \geqslant c \mu>0$. By comparison, we conclude using (3.5) and [14, proposition 4.10]. Let $v=K_{\alpha}(w)$. We have that $v$ satisfies analogous properties to $w$, but for the inversion variable

$$
\left.\begin{array}{ll}
v(X) \geqslant \varepsilon & \text { in } B_{1}^{+}, \\
v(X) \leqslant \mu|X|^{\alpha-N} & \text { in } \mathbb{R}_{+}^{N+1} \backslash B_{1}^{+},
\end{array}\right\}
$$

as well as being a solution to the problem

$$
\left.\begin{array}{rlrl}
-L_{\alpha} v & =0 & & \text { in } \mathbb{R}_{+}^{N+1}, \\
\frac{\partial v}{\partial \nu^{\alpha}} & =|x|^{-\gamma} v^{p} & & \text { for } y=0,|x| \neq 0,
\end{array}\right\}
$$

where $\gamma=(N+\alpha)-(N-\alpha) p>0$.

We now proceed with the reflection. Let

$$
\psi_{\rho}(X)=v\left(X^{\rho}\right)-v(X) .
$$

Clearly, $L_{\alpha}\left(\psi_{\rho}\right)=0$ in $\mathbb{R}_{+}^{N+1}$. We want to prove that $\psi_{\rho} \geqslant 0$ in $\tilde{\Sigma}_{\rho}$. Recall that $v$ may have a singularity at the origin, and therefore $\psi_{\rho}$ may have a singularity at $P_{\rho}$. We begin with the following result. 
LEMMA 3.2. With the above notation, we have $\psi_{\rho} \geqslant 0$ in $\tilde{\Sigma}_{\rho}$, provided $\rho>0$ is sufficiently large.

Proof. Let $\beta>0$ be some constant to be chosen later, and let

$$
\varphi_{\rho}(X)=|Z|^{\beta} \psi_{\rho}(X), \quad Z=X+e_{N+1}=(x, y+1) .
$$

From equation (3.7), we obtain

$$
L_{\alpha}\left(\varphi_{\rho}\right)-\beta y^{1-\alpha}|Z|^{-2}\left[(-\beta+N-\alpha) \varphi_{\rho}+2\left\langle Z, \nabla \varphi_{\rho}\right\rangle\right]=0 .
$$

Assume by contradiction that there exists $\delta>0$ such that

$$
\inf _{\tilde{\Sigma}_{\rho}} \varphi_{\rho}=-\delta<0
$$

First of all, we observe that (3.6) implies

$$
\left|\varphi_{\rho}\right| \leqslant c|X|^{\beta+\alpha-N} \rightarrow 0 \text { for }|X| \rightarrow \infty,
$$

if we take $\beta<N-\alpha$. On the other hand, close to the possible singularity $P_{\rho}$, we have $\varphi_{\rho}>0$. In fact, if $X \in B_{1}^{+}\left(P_{\rho}\right)$, then $X^{\rho} \in B_{1}^{+}$, and then $v\left(X^{\rho}\right) \geqslant \varepsilon$. Since $v(X) \leqslant \mu|X|^{\alpha-N} \leqslant \mu \rho^{\alpha-N}$, we obtain

$$
\varphi_{\rho}(X) \geqslant|Z|^{\beta}\left(\varepsilon-\mu|\rho|^{\alpha-N}\right)>0 \quad \text { in } B_{1}^{+}\left(P_{\rho}\right),
$$

provided $\rho$ is sufficiently large. Therefore, the infimum in (3.11) is achieved in a point of regularity of $\varphi_{\rho}$. As for the interior points, the above choice of $\beta$ gives that equation (3.10) does not allow for interior minima to exist. Finally, the fact that $\varphi_{\rho}=0$ on $T_{\rho}$ leads to the only possibility of the infimum being achieved, namely, on the part of the boundary $\Sigma_{\rho} \cap\{y=0\}$. Then let $\left(x_{0}, 0\right) \in \Sigma_{\rho} \cap\{y=0\}$ be such that $\varphi_{\rho}\left(x_{0}, 0\right)=-\delta$.

We claim that the boundary condition in (3.7) implies

$$
\frac{\partial \varphi_{\rho}}{\partial \nu^{\alpha}}\left(x_{0}\right)>0
$$

which will give the desired contradiction. It is at this point that the condition $\alpha \geqslant 1$ enters.

By Leibniz's rule, we have

$$
\frac{\partial \varphi_{\rho}}{\partial \nu^{\alpha}}\left(x_{0}\right)=\left|\left(x_{0}, 1\right)\right|^{\beta} \frac{\partial \psi_{\rho}}{\partial \nu^{\alpha}}\left(x_{0}\right)+\psi_{\rho}\left(x_{0}, 0\right) \frac{\partial|Z|^{\beta}}{\partial \nu^{\alpha}}\left(x_{0}\right) .
$$

The first term is bounded below, since, by using (3.6), (3.7) and the mean value theorem, we obtain

$$
\begin{aligned}
\frac{\partial \psi_{\rho}}{\partial \nu^{\alpha}}\left(x_{0}\right) & =\left|x_{0}^{\rho}\right|^{-\gamma} v^{p}\left(x_{0}^{\rho}, 0\right)-\left|x_{0}\right|^{-\gamma} v^{p}\left(x_{0}, 0\right) \\
& \geqslant\left|x_{0}\right|^{-\gamma}\left(v^{p}\left(x_{0}^{\rho}, 0\right)-v^{p}\left(x_{0}, 0\right)\right) \\
& \geqslant p\left|x_{0}\right|^{-\gamma} v^{p-1}\left(x_{0}, 0\right) \psi_{\rho}\left(x_{0}, 0\right),
\end{aligned}
$$

and thus

$$
\left|\left(x_{0}, 1\right)\right|^{\beta} \frac{\partial \psi_{\rho}}{\partial \nu^{\alpha}}\left(x_{0}\right) \geqslant-p \delta\left|x_{0}\right|^{-\gamma-(p-1)(N-\alpha)} \geqslant-c \rho^{-2} .
$$


For the second term,

$$
\frac{\partial|Z|^{\beta}}{\partial \nu^{\alpha}}\left(x_{0}\right)= \begin{cases}0 & \text { if } \alpha<1 \\ -\beta\left|\left(x_{0}, 1\right)\right|^{\beta-2} & \text { if } \alpha=1 \\ -\infty & \text { if } \alpha>1\end{cases}
$$

For the case where $\alpha>1$, we conclude that $\partial \varphi_{\rho} / \partial \nu^{\alpha}\left(x_{0}\right)=+\infty$. In the case where $\alpha=1$, a sharp control of the above terms gives (3.12); this is done in [32]. In the case where $\alpha<1$, the condition (3.12) is not necessarily true.

The moving planes method begins with a plane in which we find some kind of symmetry and then we see how far this plane can be moved while keeping that symmetry. The above lemma, instrumental in unbounded domains, provides a 'starting plane'. The following lemma establishes that we can move that plane up to the origin.

Lemma 3.3. Let $\rho_{0}$ be defined as

$$
\rho_{0}=\inf \left\{\rho>0 ; \varphi_{\mu} \geqslant 0 \text { in } \tilde{\Sigma_{\mu}} \text { for all } \rho<\mu<\infty\right\} .
$$

Then $\rho_{0}=0$.

Proof. By lemma 3.2, $\rho_{0}$ is finite. Suppose that $\rho_{0}>0$. By continuity, we have $\varphi_{\rho_{0}}=|z|^{\beta} \psi_{\rho_{0}} \geqslant 0$ in $\tilde{\Sigma}_{\rho_{0}}$. Since $\gamma>0$ and $\rho_{0}>0$, we have by the boundary condition that $\psi_{\rho_{0}} \not \equiv 0$ in $\tilde{\Sigma}_{\rho_{0}}$. Also, by (3.13), $\partial \psi_{\rho_{0}} / \partial \nu^{\alpha} \geqslant 0$ on $\{y=0\} \cap \bar{\Sigma}_{\rho_{0}}$. Clearly, $L_{\alpha}\left(\psi_{\rho_{0}}\right)=0$ in $\mathbb{R}_{+}^{N+1}$ and, in particular, in the set

$$
R_{0}=\left\{\left|X-P_{\rho_{0}}\right|=\frac{1}{2}\left|\rho_{0}\right|, y \geqslant 0\right\} .
$$

Therefore, by proposition 4.10 of [14], we have $\psi_{\rho_{0}}>0$ in $R_{0}$. Let $\delta=\inf _{R_{0}} \psi_{\rho_{0}}>0$. The function $\psi_{\rho_{0}}$ may have a singularity at $P_{\rho_{0}}$, so we construct the following auxiliary function. Let $h_{\varepsilon}$ be the solution to the problem

$$
\left.\begin{array}{rlrlrl}
L_{\alpha}\left(h_{\varepsilon}\right)(X) & =0, & \varepsilon<\left|X-P_{\rho_{0}}\right| & <\frac{1}{2}\left|\rho_{0}\right|, & & y>0, \\
h_{\varepsilon}(X) & =\delta, & \left|X-P_{\rho_{0}}\right| & =\frac{1}{2}\left|\rho_{0}\right|, & & y \geqslant 0, \\
h_{\varepsilon}(X) & =0, & \left|X-P_{\rho_{0}}\right| & =\varepsilon, & & y \geqslant 0, \\
\frac{\partial h_{\varepsilon}}{\partial \nu^{\alpha}}(X) & =0, & \varepsilon<\left|X-P_{\rho_{0}}\right| & <\frac{1}{2}\left|\rho_{0}\right|, & & y=0 .
\end{array}\right\}
$$

Then lemma 4.11 of [14] implies

$$
\psi_{\rho_{0}} \geqslant h_{\varepsilon} \quad \text { in } \varepsilon \leqslant\left|X-P_{\rho_{0}}\right| \leqslant \frac{1}{2}\left|\rho_{0}\right|, \quad y \geqslant 0
$$

Letting $\varepsilon \rightarrow 0^{+}$we have $\lim _{\varepsilon \rightarrow 0^{+}} h_{\varepsilon} \equiv \delta$ by the uniqueness of the $\alpha$-harmonic extension. Therefore,

$$
\psi_{\rho_{0}} \geqslant \delta \quad \text { in } 0<\left|X-P_{\rho_{0}}\right| \leqslant \frac{1}{2}\left|\rho_{0}\right|, \quad y \geqslant 0 .
$$

Since $\varphi_{\rho_{0}} \geqslant \psi_{\rho_{0}}$ in $\tilde{\Sigma}_{\rho_{0}}$, we have

$$
\lim _{\rho \rightarrow \rho_{0}} \inf _{R_{0}} \varphi_{\rho} \geqslant \inf _{R_{0}} \varphi_{\rho_{0}} \geqslant \delta .
$$


If $\rho_{0}$ is the infimum, there exists a sequence $\rho_{k} \nearrow \rho_{0}$ such that

$$
\inf _{\tilde{\Sigma}_{\rho_{k}}} \varphi_{\rho_{k}}<0 .
$$

Clearly, $\lim _{|X| \rightarrow \infty} \varphi_{\rho_{k}}=0$. Recalling (3.18) the infimum in (3.19) must be attained at some finite point $X^{k} \in \bar{\Sigma}_{\rho_{k}} \backslash B_{\left|\rho_{0}\right| / 2}\left(P_{\rho_{0}}\right)$ with sufficiently small $\left|\rho_{k}-\rho_{0}\right|$. On the other hand, $X^{k} \notin T_{\rho_{k}}$, since $\varphi_{\rho_{k}} \equiv 0$ in $T_{\rho_{k}}$. Therefore, $X^{k}$ must belong to the set

$$
\left\{X \in \mathbb{R}^{N+1} ; y=0, x_{N}>0,\left|X-P_{\rho_{0}}\right|^{2} \geqslant \frac{1}{4}\left|\rho_{0}\right|^{2}\right\} .
$$

Reasoning as in lemma 3.2 , this leads to the desired contradiction.

Now we can deal with the proof of the main theorem in this subsection.

Proof of theorem 3.1. Let $w$ be any solution to problem (3.1) and consider its fractional Kelvin transform $v=K_{\alpha}(w)$. By lemma (3.3) we have

$$
v\left(x_{1}, \ldots, x_{N}, y\right) \geqslant v\left(x_{1}, \ldots,-x_{N}, y\right) \text { for } x_{N}>0 .
$$

The same argument fits for negative $x_{N}$ giving the reverse inequality. Therefore, $v(X)$ is symmetric with respect to the $x_{N}$-axis. Obviously, we can apply this argument in every direction perpendicular to $y$-axis. Hence, $v(X)$ is a two-variable function, as is $w(X)$. Indeed,

$$
w(X)=\phi(|x|, y)
$$

for some function $\phi$. Hence, $w$ is independent of $\left(x_{1}, \ldots, x_{N}\right)$, and therefore $w(X)=$ $w(y)$.

To complete the proof we consider the problem in one dimension.

$$
\left.\begin{array}{rl}
-\left(y^{1-\alpha} w^{\prime}\right)^{\prime} & =0 \quad \text { for } y>0, \\
\lim _{y \rightarrow 0^{+}} y^{1-\alpha} w^{\prime}(y) & =w^{p}(0) .
\end{array}\right\}
$$

The solutions of this problem are of the form $w(y)=c-c^{p} / \alpha y^{\alpha}$ with $c \geqslant 0$, which implies that the only non-negative solution is $w \equiv 0$.

\subsection{A problem in a quarter-space}

THEOREM 3.4. Let $1 \leqslant \alpha<2$. Then the problem in the first quarter

$$
\mathbb{R}_{++}^{N+1}=\left\{X=\left(x^{\prime}, x_{N}, y\right) \mid x^{\prime} \in \mathbb{R}^{N-1}, x_{N}>0, y>0\right\}
$$

and

$$
\left.\begin{array}{rl}
-\operatorname{div}\left(y^{1-\alpha} \nabla w\right) & =0, \quad x_{N}>0, y>0, \\
\frac{\partial w}{\partial \nu^{\alpha}}\left(x^{\prime}, x_{N}\right) & =w^{p}\left(x^{\prime}, x_{N}, 0\right), \\
w\left(x^{\prime}, 0, y\right) & =0,
\end{array}\right\}
$$

has no positive bounded solution, provided that $p<(N+\alpha) /(N-\alpha)$.

Theorem 3.4 is proved in the case $\alpha=1$ in [15]. We begin with a generalization of proposition 6.1 of [21]. 
LEMMA 3.5. Suppose $w$ is a solution of the following problem:

$$
\left.\begin{array}{c}
-L_{\alpha} w \geqslant 0, \quad w \geqslant 0 \quad \text { in } \mathbb{R}_{+}^{2} \\
\frac{\partial w}{\partial \nu^{\alpha}} \geqslant 0 \quad \text { for } y=0, \quad
\end{array}\right\}
$$

then $w$ is a constant.

Proof. Let $X_{0}=\left(x_{0}, y_{0}\right) \in \overline{\mathbb{R}_{+}^{2}}$. Given $\varepsilon, \delta \in(0,1)$, we define the function

$$
\psi(X)=\varepsilon w\left(X_{0}\right) \log \left(\frac{\left|X-X_{0}\right|^{2}}{\delta^{2}}\right)+C_{\delta},
$$

where

$$
C_{\delta}=\frac{\max }{S_{\delta}^{+}\left(X_{0}\right)}\left(w\left(X_{0}\right)-w(X)\right),
$$

where

$$
\overline{S_{\delta}^{+}\left(X_{0}\right)}=\left\{\left|X-X_{0}\right|=\delta, y \geqslant 0\right\}
$$

It is clear that

$$
\psi(X) \equiv C_{\delta} \quad \text { on } \overline{S_{\delta}^{+}\left(X_{0}\right)}
$$

and, taking sufficiently small $\delta$, we have

$$
\psi(X) \geqslant w\left(X_{0}\right) \geqslant w\left(X_{0}\right)-w(X) \quad \text { on } \overline{S_{\mathrm{e}^{1 / \varepsilon}}^{+}\left(X_{0}\right)}
$$

A direct calculation shows that if $\alpha \in(1,2)$, then

$$
\begin{aligned}
-L_{\alpha} \psi \leqslant 0 & \text { in } \mathbb{R}_{+}^{2}, \\
\frac{\partial \psi}{\partial \nu^{\alpha}}=\infty & \text { for } y=0 .
\end{aligned}
$$

Thus, by the maximum principle,

$$
\psi(X) \geqslant w\left(X_{0}\right)-w(X) \quad \text { for } X \in \overline{\mathbb{R}_{+}^{2}}, \quad \delta<\left|X-X_{0}\right|<\mathrm{e}^{1 / \varepsilon} .
$$

Letting $\varepsilon \rightarrow 0$ and then $\delta \rightarrow 0$, we have $w\left(X_{0}\right)-w(X) \leqslant 0$ for any $X_{0}, X \in \overline{\mathbb{R}_{+}^{2}}$.

Lemma 3.6. Let $p \geqslant 0$ and let $C$ be a positive constant. Then there is no solution to the problem

$$
\left.\begin{array}{rlrl}
-L_{\alpha} w & =0, & & 0<w \leqslant C \text { in } \mathbb{R}_{++}^{2}=\{x>0, y>0\}, \\
\frac{\partial w}{\partial \nu^{\alpha}} & =w^{p} & & \text { on }\{x>0, y=0\}, \\
w & =0 & & \text { on }\{x=0, y \geqslant 0\} .
\end{array}\right\}
$$

Proof. First, we show that $w(x, 0) \rightarrow 0$ as $x \rightarrow \infty$. Suppose, by contradiction, that there exists a sequence $\eta_{m} \rightarrow \infty$ as $m \rightarrow \infty$ and such that $w\left(\eta_{m}, 0\right) \rightarrow K>0$. Let us define $w_{m}(x, y)=w\left(x+\eta_{m}, y\right)$. It clearly holds that

$$
\begin{aligned}
& -L_{\alpha} w_{m}=0, \quad 0<w_{m} \leqslant C \text { in } R_{m}=\left\{x>\eta_{m}, y>0\right\}, \\
& \frac{\partial w_{m}}{\partial \nu^{\alpha}}=w^{p} \quad \text { on }\left\{x>\eta_{m}, y=0\right\} \text {, } \\
& w_{m}=0 \quad \text { on }\left\{x=\eta_{m}, y \geqslant 0\right\} \text {. }
\end{aligned}
$$


Moreover, $w_{m}(0,0) \rightarrow K$. So that, taking a subsequence of $w_{m}$ if necessary, we have $w_{m} \rightarrow \tilde{w}$ with

$$
\left.\begin{array}{rlrl}
-L_{\alpha} \tilde{w} & =0, & & 0 \leqslant \tilde{w} \leqslant C \text { in } \mathbb{R}_{+}^{2}, \\
\frac{\partial \tilde{w}}{\partial \nu^{\alpha}} & =\tilde{w}^{p} \geqslant 0 & & \text { for } y=0 .
\end{array}\right\}
$$

Since $\tilde{w}(0,0)=K$, lemma 3.5 implies $\tilde{w} \equiv K$, but by the boundary condition we have that

$$
\frac{\partial \tilde{w}}{\partial \nu^{\alpha}}(0,0)=\tilde{w}^{p}(0,0)=K^{p}>0,
$$

which leads to a contradiction. Therefore, $w(x, 0) \rightarrow 0$ as $x \rightarrow \infty$.

Following [14], we define the function

$$
\Phi(x)=\frac{1}{2} \int_{0}^{\infty} y^{1-\alpha}\left(\left|w_{x}(x, y)\right|^{2}-\left|w_{y}(x, y)\right|^{2}\right) \mathrm{d} y,
$$

see also [15] for the case where $\alpha=1$. Differentiating inside the integral, we have

$$
\frac{1}{2} \int_{0}^{\infty} \frac{\partial}{\partial x}\left[y^{1-\alpha}\left(\left|w_{x}\right|^{2}-\left|w_{y}\right|^{2}\right)\right] \mathrm{d} y=\int_{0}^{\infty} y^{1-\alpha}\left(w_{x x} w_{x}-w_{y} w_{x y}\right) \mathrm{d} y .
$$

We want to see that this integral converges. By lemma 4.3 of [14], we know that there exists some $\beta \in(0,1)$ such that $w \in C^{2, \beta}$. Moreover, by proposition 4.6 of [14],

$$
\begin{aligned}
\int_{0}^{\infty} y^{1-\alpha}\left(\left|w_{x x} w_{x}\right|+\left|w_{y} w_{x y}\right|\right) \mathrm{d} y \\
\quad \leqslant M_{1}\left(\int_{0}^{1} y^{1-\alpha}\left(\left|w_{x}\right|+\left|w_{y}\right|\right) \mathrm{d} y+\int_{1}^{\infty} y^{1-\alpha}\left(\left|w_{x}\right|+\left|w_{y}\right|\right) \mathrm{d} y\right) \\
\quad \leqslant M_{2}\left(M_{3}+\int_{1}^{\infty} \frac{y^{1-\alpha}}{y+1} \mathrm{~d} y\right) \\
\quad<\infty
\end{aligned}
$$

for some constants $M_{1}, M_{2}, M_{3}>0$. Note that the last integral is convergent provided $1<\alpha<2$. We recall that, in the case where $\alpha=1$, a sharper estimate is used in [15]. Now let

$$
G(w)=\int_{0}^{w} f(s) \mathrm{d} s .
$$

By dominated convergence, and since $|\nabla w(x, y)| \rightarrow 0$ as $y \rightarrow \infty$, integrating by parts yields

$$
\begin{aligned}
{[\Phi(x)+G(w(x, 0))]_{x} } & =\int_{0}^{\infty} y^{1-\alpha}\left[w_{x x} w_{x}-w_{y} w_{x y}\right](x, y) \mathrm{d} y+\left[f(w) w_{x}\right](x, 0) \\
& =\lim _{y \rightarrow 0}\left[y^{1-\alpha} w_{y} w_{x}+f(w) w_{x}\right](x, y) \\
& =\lim _{y \rightarrow 0}\left[y^{1-\alpha} w_{y} w_{x}-y^{1-\alpha} w_{y} w_{x}\right](x, y) \\
& =0 .
\end{aligned}
$$


Therefore, $\Phi(x)+G(w(x, 0))$ is constant. The rest of the proof is exactly the same as in [15]. Using that $w(x, 0) \rightarrow 0$ as $x \rightarrow \infty$ and lemma 5.1 of [14], we obtain

$$
\Phi(x)+G(w(x, 0)) \equiv 0 .
$$

Since $w \equiv 0$ in $\{x=0, y \geqslant 0\}$, it follows that

$$
0=2 \Phi(0)=\int_{0}^{\infty}\left|w_{x}\right|^{2}(0, y) \mathrm{d} y
$$

which implies $w_{x}=0$ on $\{x=0, y>\varepsilon\}$ for every $\varepsilon>0$. Since $L_{\alpha}$ is a nondegenerated elliptic operator in $\{x=0, y>\varepsilon\}$, by Hopf's lemma this leads to a contradiction.

With these two results, a standard argument completes the proof.

Proof of theorem 3.4. By an analogous argument to the proof of theorem 3.4 for the $\left(x_{1}, \ldots, x_{N-1}\right)$ variables (with the analogous lemmas 3.2 and 3.3 ), it is easy to see that any positive solution of (3.23) depends only on two variables: $x_{N}$ and $y$. Therefore, applying proposition 3.6, the proof is complete.

\section{The fractional Laplacian in a bounded domain}

\subsection{The $\alpha$-harmonic extension}

To define the fractional Laplacian in a bounded domain we follow [15] (see also [41]). The idea is to use the $\alpha$-harmonic extension introduced in [17] to define the same operator in the whole space, but restricted to our bounded domain. To this end, we consider the cylinder

$$
C_{\Omega}=\left\{(x, y): x \in \Omega, y \in \mathbb{R}_{+}\right\} \subset \mathbb{R}_{+}^{N+1},
$$

and denote by $\partial_{\mathrm{L}} C_{\Omega}$ its lateral boundary.

We first define the extension operator and fractional Laplacian for smooth functions.

Definition 4.1. Given a regular function $u$, we define its $\alpha$-harmonic extension $w=E_{\alpha}(u)$ to the cylinder $C_{\Omega}$ as the solution to the problem

$$
\left.\begin{array}{rl}
-\operatorname{div}\left(y^{1-\alpha} \nabla w\right)=0 & \text { in } C_{\Omega}, \\
w=0 & \text { on } \partial_{\mathrm{L}} C_{\Omega}, \\
w=u & \text { on } \Omega .
\end{array}\right\}
$$

As in the whole space, there is also a Poisson formula for the extension operator in a bounded domain, defined through the Laplace transform and the heat semigroup generator $\mathrm{e}^{t \Delta}$ (see [41] for details).

Definition 4.2. The fractional operator $(-\Delta)^{\alpha / 2}$ in $\Omega$, acting on a regular function $u$, is defined by

$$
(-\Delta)^{\alpha / 2} u(x)=-\frac{1}{\kappa_{\alpha}} \lim _{y \rightarrow 0^{+}} y^{1-\alpha} \frac{\partial w}{\partial y}(x, y),
$$

where $w=E_{\alpha}(u)$ and $\kappa_{\alpha}$ is given as in (2.1). 
This operator can be extended by density to a fractional Sobolev space.

\subsection{Spectral decomposition}

It is classical that the powers of a positive operator in a bounded domain (or in an unbounded domain, provided the spectrum is discrete) are defined through the spectral decomposition using the powers of the eigenvalues of the original operator. We show next that, in this case, this is coherent with the Dirichlet-Neumann operator defined above. Let $\left(\varphi_{j}, \lambda_{j}\right)$ be the eigenfunctions and eigenvectors of $-\Delta$ in $\Omega$ with Dirichlet boundary data. Define the space of functions defined in our domain $\Omega$ as

$$
H_{0}^{\alpha / 2}(\Omega)=\left\{u=\sum a_{j} \varphi_{j} \in L^{2}(\Omega):\|u\|_{H_{0}^{\alpha / 2}}=\left(\sum a_{j}^{2} \lambda_{j}^{\alpha / 2}\right)^{1 / 2}<\infty\right\},
$$

its topological dual as $H^{-\alpha / 2}(\Omega)$, and the energy space $X_{0}^{\alpha}\left(C_{\Omega}\right)$ of functions defined in the cylinder $C_{\Omega}$ as the completion of $C_{0}^{\infty}(\Omega \times[0, \infty))$ with the norm

$$
\|z\|_{X_{0}^{\alpha}}=\left(\int_{C_{\Omega}} y^{1-\alpha}|\nabla z|^{2}\right)^{1 / 2} .
$$

Lemma 4.3 .

(i) The eigenfunctions and eigenvectors of $(-\Delta)^{\alpha / 2}$ in $\Omega$ with Dirichlet boundary data are given by $\left(\varphi_{j}, \lambda_{j}^{\alpha / 2}\right)$.

(ii) If

$$
u=\sum a_{j} \varphi_{j} \in H_{0}^{\alpha / 2}(\Omega)
$$

then $E_{\alpha}(u) \in X_{0}^{\alpha}\left(C_{\Omega}\right)$ and

$$
E_{\alpha}(u)(x, y)=\sum a_{j} \varphi_{j}(x) \psi\left(\lambda_{j}^{1 / 2} y\right),
$$

where $\psi(s)$ solves the problem

$$
\begin{aligned}
\psi^{\prime \prime}+\frac{1-\alpha}{s} \psi^{\prime} & =\psi, \quad s>0, \\
-\lim _{s \rightarrow 0^{+}} s^{1-\alpha} \psi^{\prime}(s) & =\kappa_{\alpha}, \\
\psi(0) & =1 .
\end{aligned}
$$

(iii) In the same hypotheses, $(-\Delta)^{\alpha / 2} u \in H^{-\alpha / 2}(\Omega)$, and

$$
(-\Delta)^{\alpha / 2} u=\sum a_{j} \lambda_{j}^{\alpha / 2} \varphi_{j}
$$

(iv) It holds that

$$
\left\|(-\Delta)^{\alpha / 2} u\right\|_{H^{-\alpha / 2}}=\left\|(-\Delta)^{\alpha / 4} u\right\|_{L^{2}}=\|u\|_{H_{0}^{\alpha / 2}}=\kappa_{\alpha}^{-1 / 2}\left\|E_{\alpha}(u)\right\|_{X_{0}^{\alpha}} .
$$


The proof of this result is straightforward. The function $\psi$ coincides with the solution $\phi_{\alpha}$ in problem (2.8). The calculation of the norms is also straightforward. Using the orthogonality of the family $\left\{\varphi_{j}\right\}$, together with $\int_{\Omega} \varphi_{j}^{2}=1, \int_{\Omega}\left|\nabla \varphi_{j}\right|^{2}=\lambda_{j}$ and (2.10), we have

$$
\begin{aligned}
\int_{C_{\Omega}} & y^{1-\alpha}\left|\nabla E_{\alpha}(u)(x, y)\right|^{2} \mathrm{~d} x \mathrm{~d} y \\
& =\int_{0}^{\infty} y^{1-\alpha} \int_{\Omega}\left(\sum a_{j}^{2}\left|\nabla \varphi_{j}(x)\right|^{2} \psi\left(\lambda_{j}^{1 / 2} y\right)^{2}+a_{j}^{2} \lambda_{j} \varphi_{j}(x)^{2}\left(\psi^{\prime}\left(\lambda_{j}^{1 / 2} y\right)\right)^{2}\right) \mathrm{d} x \mathrm{~d} y \\
& =\int_{0}^{\infty} y^{1-\alpha} \sum a_{j}^{2} \lambda_{j}\left(\psi\left(\lambda_{j}^{1 / 2} y\right)^{2}+\left(\psi^{\prime}\left(\lambda_{j}^{1 / 2} y\right)\right)^{2}\right) \mathrm{d} y \\
& =\sum a_{j}^{2} \lambda_{j}^{\alpha / 2} \int_{0}^{\infty} s^{1-\alpha}\left(\psi(s)^{2}+\left(\psi^{\prime}(s)\right)^{2}\right) \mathrm{d} s \\
& =\kappa_{\alpha} \sum a_{j}^{2} \lambda_{j}^{\alpha / 2} \\
& =\kappa_{\alpha} \sum\left(a_{j} \lambda_{j}^{\alpha / 4}\right)^{2} .
\end{aligned}
$$

\subsection{The trace inequality}

Using the trace inequality in the whole space (2.4), we obtain the corresponding inequality for bounded domains. To do this, we consider $v \in X_{0}^{\alpha}\left(C_{\Omega}\right)$. Its extension by zero outside the cylinder $C_{\Omega}$ can be approximated by functions with compact support in $\overline{\mathbb{R}_{+}^{N+1}}$. Thus, the trace inequality (2.4), together with Hölder's inequality, gives a trace inequality for bounded domains.

TheOREM 4.4. For any $1 \leqslant r \leqslant 2 N /(N-\alpha)$, and every $z \in X_{0}^{\alpha}\left(C_{\Omega}\right)$, it holds that

$$
\left(\int_{\Omega}|v(x)|^{r} \mathrm{~d} x\right)^{2 / r} \leqslant C(r, \alpha, N,|\Omega|) \int_{C_{\Omega}} y^{1-\alpha}|\nabla z(x, y)|^{2} \mathrm{~d} x \mathrm{~d} y,
$$

where $v=\operatorname{tr}(z)$.

\subsection{The linear problem}

We now use the extension problem (4.1) and the expression (4.2) to reformulate the non-local problems in a local way. Let $g$ be a regular function and consider the following problems: the non-local problem

$$
\left.\begin{array}{rlr}
(-\Delta)^{\alpha / 2} u & =g(x) & \text { in } \Omega \\
u & =0 & \text { on } \partial \Omega
\end{array}\right\}
$$

and the corresponding local one

$$
\left.\begin{array}{rlrl}
-\operatorname{div}\left(y^{1-\alpha} \nabla w\right) & =0 & & \text { in } C_{\Omega}, \\
w & =0 & & \text { on } \partial_{\mathrm{L}} C_{\Omega}, \\
-\frac{1}{\kappa} \lim _{\alpha \rightarrow 0^{+}} y^{1-\alpha} \frac{\partial w}{\partial y} & =g(x) & & \text { on } \Omega .
\end{array}\right\}
$$


We want to define the concept of the solution to (4.4), which is done in terms of the solution to problem (4.5).

Definition 4.5. We say that $w \in X_{0}^{\alpha}\left(C_{\Omega}\right)$ is an energy solution to problem (4.5) if, for every function $\varphi \in \mathcal{C}_{0}^{1}\left(C_{\Omega}\right)$, it holds that

$$
\int_{C_{\Omega}} y^{1-\alpha}\langle\nabla w(x, y), \nabla \varphi(x, y)\rangle \mathrm{d} x \mathrm{~d} y=\int_{\Omega} \kappa_{\alpha} g(x) \varphi(x, 0) \mathrm{d} x .
$$

In fact, more general test functions can be used in the above formula whenever the integrals make sense. A supersolution (respectively, subsolution) is a function that verifies $(4.6)$ with equality replaced by ' $\geqslant$ ' (respectively, ' $\leqslant$ ') for every non-negative test function.

Definition 4.6. We say that $u \in H_{0}^{\alpha / 2}(\Omega)$ is an energy solution to problem (4.4) if it is the trace on $\Omega$ of a function $w$ that is an energy solution to problem (4.5).

It is clear that a solution exists, for example, for every $g \in H^{-\alpha / 2}(\Omega)$. In order to deal with problem (4.5) we shall assume, without loss of generality, that $\kappa_{\alpha}=1$, by changing the function $g$.

This linear problem is also mentioned in [14]. There, some results are obtained using the theory of degenerate elliptic equations developed in [26]. In particular, a regularity result for bounded solutions to this problem is obtained in [14]. We prove here that the solutions are in fact bounded if $g$ satisfies a minimal integrability condition.

Theorem 4.7. Let $w$ be a solution to problem (4.5). If $g \in L^{r}(\Omega), r>N / \alpha$, then $w \in L^{\infty}\left(C_{\Omega}\right)$.

Proof. The proof follows from the well-known Moser iterative technique, which we take from [30, theorem 8.15], and uses the trace inequality (4.3). Without loss of generality, we may assume $w \geqslant 0$, and this simplifies notation. The general case is obtained in a similar way.

We define, for $\beta \geqslant 1$ and $K \geqslant k$ ( $k$ to be chosen later), a $\mathcal{C}^{1}([k, \infty))$ function $H$ as follows:

$$
H(z)= \begin{cases}z^{\beta}-k^{\beta}, & z \in[k, K], \\ \text { linear, } & z>K .\end{cases}
$$

Let us also define $v=w+k, \nu=\operatorname{tr}(v)$, and choose as test function $\varphi$ :

$$
\begin{aligned}
\varphi & =G(v)=\int_{k}^{v}\left|H^{\prime}(s)\right|^{2} \mathrm{~d} s, \\
\nabla \varphi & =\left|H^{\prime}(v)\right|^{2} \nabla v .
\end{aligned}
$$

Observe that it is an admissible test function, although it is not $\mathcal{C}^{1}$. Substituting this test function into the definition of the energy solution, we obtain, on the one 
hand

$$
\begin{aligned}
\int_{C_{\Omega}} y^{1-\alpha}\langle\nabla w, \nabla \varphi\rangle \mathrm{d} x \mathrm{~d} y & =\int_{C_{\Omega}} y^{1-\alpha}|\nabla v|^{2}\left|H^{\prime}(v)\right|^{2} \mathrm{~d} x \mathrm{~d} y \\
& =\int_{C_{\Omega}} y^{1-\alpha}|\nabla H(v)|^{2} \mathrm{~d} x \mathrm{~d} y \\
& \geqslant\left(\int_{\Omega}|H(\nu)|^{2 N /(N-\alpha)} \mathrm{d} x\right)^{(N-\alpha) / N} \\
& =\|H(\nu)\|_{2 N /(N-\alpha)}^{2},
\end{aligned}
$$

where the last inequality follows by (4.3), and, on the other hand,

$$
\begin{aligned}
\int_{\Omega} g(x) \varphi(x, 0) \mathrm{d} x & =\int_{\Omega} g(x) G(\nu) \mathrm{d} x \\
& \leqslant \int_{\Omega} g(x) \nu G^{\prime}(\nu) \mathrm{d} x \\
& \leqslant \frac{1}{k} \int_{\Omega} g(x) \nu^{2}\left|H^{\prime}(\nu)\right|^{2} \mathrm{~d} x \\
& =\frac{1}{k} \int_{\Omega} g(x)\left|\nu H^{\prime}(\nu)\right|^{2} \mathrm{~d} x .
\end{aligned}
$$

Inequality (4.7), together with (4.8), leads to

$$
\|H(\nu)\|_{2 N /(N-\alpha)} \leqslant\left(\frac{1}{k}\|g\|_{r}\right)^{1 / 2}\left\|\left(\nu H^{\prime}(\nu)\right)^{2}\right\|_{r /(r-1)}^{1 / 2}=\left\|\nu H^{\prime}(\nu)\right\|_{2 r /(r-1)},
$$

by choosing $k=\|g\|_{r}$. Letting $K \rightarrow \infty$ in the definition of $H$, inequality (4.9) becomes

$$
\|\nu\|_{2 N \beta /(N-\alpha)} \leqslant\|\nu\|_{2 r \beta /(r-1)} .
$$

Hence, for all $\beta \geqslant 1$, the inclusion $\nu \in L^{2 r \beta /(r-1)}(\Omega)$ implies the stronger inclusion $\nu \in L^{2 N \beta /(N-\alpha)}(\Omega)$, since $2 N \beta /(N-\alpha)>2 r \beta /(r-1)$ provided $r>N / \alpha$. The result now follows, as in [30], by an iteration argument, starting with

$$
\beta=\frac{N(r-1)}{r(N-\alpha)}>1 \quad \text { and } \quad \nu \in L^{2 N /(N-\alpha)}(\Omega) .
$$

This gives $\nu \in L^{\infty}(\Omega)$, and then $w \in L^{\infty}\left(C_{\Omega}\right)$. In fact, we get the estimate

$$
\|w\|_{\infty} \leqslant c\left(\|w\|_{X^{\alpha}}+\|g\|_{r}\right) .
$$

Corollary 4.8. Let $w$ be a solution to problem (4.5). If $g \in L^{\infty}(\Omega)$, then $w \in$ $\mathcal{C}^{\gamma}\left(\bar{C}_{\Omega}\right)$ for some $\gamma \in(0,1)$.

Proof. Using theorem 4.7, the result follows directly from [14, lemma 4.4], where it is proved that any bounded solution to problem (4.5) with a bounded $g$ is $\mathcal{C}^{\gamma}$. 


\section{The nonlinear non-local problem}

\subsection{The local realization}

We deal now with the core of the paper, i.e. the study of the non-local problem (1.1). We write that problem in the local version as follows. A solution to problem (1.1) is a function $u=\operatorname{tr}(w)>0$, the trace of $w$ on $\Omega \times\{y=0\}$, where $w$ solves the local problem

$$
\left.\begin{array}{rlrl}
-\operatorname{div}\left(y^{1-\alpha} \nabla w\right) & =0 & & \text { in } C_{\Omega}, \\
w & =0 & & \text { on } \partial_{\mathrm{L}} C_{\Omega}, \\
\frac{\partial w}{\partial \nu^{\alpha}} & =f(w),, & & w>0 \text { in } \Omega,
\end{array}\right\}
$$

where

$$
\frac{\partial w}{\partial \nu^{\alpha}}(x)=-\lim _{y \rightarrow 0^{+}} y^{1-\alpha} \frac{\partial w}{\partial y}(x, y) .
$$

Note. In order to simplify notation in what follows we shall denote, when no confusion arises, $w$ for the function defined in the cylinder $C_{\Omega}$ as well as for its trace $\operatorname{tr}(w)$ on $\Omega \times\{y=0\}$. Also, in the above definition we have neglected the constant $\kappa_{\alpha}$ appearing in (4.2) by a simple rescaling.

As we have said, we shall focus on the particular nonlinearity

$$
f(s)=f_{\lambda}(s)=\lambda s^{q}+s^{p} .
$$

However many auxiliary results are proved for more general reactions $f$ satisfying the growth condition

$$
0 \leqslant f(s) \leqslant c\left(1+|s|^{p}\right) \quad \text { for some } p>0 .
$$

REMARK 5.1. According to the previous note, the results on the coefficient $\lambda$ for the local problem (5.1)-(5.3) in this section are translated into problem (1.1) with $\lambda$ multiplied by $\kappa_{\alpha}^{p(q-1)-1}$.

Following definition 4.5 , we say that $w \in X_{0}^{\alpha}\left(\mathcal{C}_{\Omega}\right)$ is an energy solution of (5.1) if the following identity holds

$$
\int_{C_{\Omega}} y^{1-\alpha}\langle\nabla w, \nabla \varphi\rangle \mathrm{d} x \mathrm{~d} y=\int_{\Omega} f(w) \varphi \mathrm{d} x
$$

for every regular test function $\varphi$. In an analogous way we define the sub- and supersolution.

We now consider the functional

$$
J(w)=\frac{1}{2} \int_{C_{\Omega}} y^{1-\alpha}|\nabla w|^{2} \mathrm{~d} x \mathrm{~d} y-\int_{\Omega} F(w) \mathrm{d} x,
$$

where

$$
F(s)=\int_{0}^{s} f(\tau) \mathrm{d} \tau
$$

For simplicity of notation, we define $f(s)=0$ for $s \leqslant 0$. Recall that the trace satisfies $w \in L^{r}(\Omega)$ (again, this means $\operatorname{tr}(w) \in L^{r}(\Omega)$ ) for every $1 \leqslant r \leqslant 2 N /(N-\alpha)$ if 
$N>\alpha$ and $1<r \leqslant \infty$ if $N \leqslant \alpha$. In particular, if $p \leqslant(N+\alpha) /(N-\alpha)$ and $f$ verifies (5.4), then $F(w) \in L^{1}(\Omega)$, and the functional is well defined and bounded from below.

It is well known that critical points of $J$ are solutions to (5.1) with a general reaction $f$. We also consider the minimization problem

$$
I=\inf \left\{\int_{C_{\Omega}} y^{1-\alpha}|\nabla w|^{2} \mathrm{~d} x \mathrm{~d} y: w \in X_{0}^{\alpha}\left(C_{\Omega}\right), \int_{\Omega} F(w) \mathrm{d} x=1\right\},
$$

for which, by classical variational techniques, below the critical exponent the infimum $I$ is achieved. This gives a non-negative solution in a standard way. Later on in the paper, we shall see that this infimum is positive provided $\lambda>0$ is sufficiently small. On the contrary, for sufficiently large $\lambda$, the infimum is the trivial solution.

We now establish two preliminary results. The first result is a classical procedure of sub- and supersolutions to obtain a solution. We omit its proof.

Lemma 5.2. Assume that there exist a subsolution $w_{1}$ and a supersolution $w_{2}$ to problem (5.1) verifying $w_{1} \leqslant w_{2}$. Then there also exists a solution $w$ satisfying $w_{1} \leqslant w \leqslant w_{2}$ in $C_{\Omega}$.

The second result is a comparison result for concave nonlinearities. The proof follows the lines of the corresponding one for the Laplacian performed in [11].

Lemma 5.3. Assume that the function $f(t) / t$ is decreasing for $t>0$ and consider $w_{1}, w_{2} \in X_{0}^{\alpha}\left(C_{\Omega}\right)$ as positive subsolution and supersolution, respectively, to problem (5.1). Then $w_{1} \leqslant w_{2}$ in $\bar{C}_{\Omega}$.

Proof. By definition we have, for the non-negative test functions $\varphi_{1}$ and $\varphi_{2}$ to be chosen in an appropriate way,

$$
\begin{aligned}
& \int_{C_{\Omega}} y^{1-\alpha}\left\langle\nabla w_{1}, \nabla \varphi_{1}\right\rangle \mathrm{d} x \mathrm{~d} y \leqslant \int_{\Omega} f\left(w_{1}\right) \varphi_{1} \mathrm{~d} x \\
& \int_{C_{\Omega}} y^{1-\alpha}\left\langle\nabla w_{2}, \nabla \varphi_{2}\right\rangle \mathrm{d} x \mathrm{~d} y \geqslant \int_{\Omega} f\left(w_{2}\right) \varphi_{2} \mathrm{~d} x .
\end{aligned}
$$

Now let $\theta(t)$ be a smooth non-decreasing function such that $\theta(t)=0$ for $t \leqslant 0$, $\theta(t)=1$ for $t \geqslant 1$, and set $\theta_{\varepsilon}(t)=\theta(t / \varepsilon)$. If, in the above inequalities, we set

$$
\varphi_{1}=w_{2} \theta_{\varepsilon}\left(w_{1}-w_{2}\right), \quad \varphi_{2}=w_{1} \theta_{\varepsilon}\left(w_{1}-w_{2}\right),
$$

we obtain

$$
I_{1} \geqslant \int_{\Omega} w_{1} w_{2}\left(\frac{f\left(w_{2}\right)}{w_{2}}-\frac{f\left(w_{1}\right)}{w_{1}}\right) \theta_{\varepsilon}\left(w_{1}-w_{2}\right) \mathrm{d} x
$$

where

$$
I_{1}:=\int_{C_{\Omega}} y^{1-\alpha}\left\langle w_{1} \nabla w_{2}-w_{2} \nabla w_{1}, \nabla\left(w_{1}-w_{2}\right)\right\rangle \theta_{\varepsilon}^{\prime}\left(w_{1}-w_{2}\right) \mathrm{d} x \mathrm{~d} y .
$$


Now we estimate $I_{1}$ as follows:

$$
\begin{aligned}
I_{1} & \leqslant \int_{\mathcal{C}_{\Omega}} y^{1-\alpha}\left\langle\nabla w_{1},\left(w_{1}-w_{2}\right) \nabla\left(w_{1}-w_{2}\right)\right\rangle \theta_{\varepsilon}^{\prime}\left(w_{1}-w_{2}\right) \mathrm{d} x \mathrm{~d} y \\
& =\int_{\mathcal{C}_{\Omega}} y^{1-\alpha}\left\langle\nabla w_{1}, \nabla \gamma_{\varepsilon}\left(w_{1}-w_{2}\right)\right\rangle \mathrm{d} x \mathrm{~d} y,
\end{aligned}
$$

where $\gamma_{\varepsilon}^{\prime}(t)=t \theta_{\varepsilon}^{\prime}(t)$. Therefore, since $0 \leqslant \gamma_{\varepsilon} \leqslant \varepsilon$, we have

$$
I_{1} \leqslant \int_{\Omega} f\left(w_{1}\right) \gamma_{\varepsilon}\left(w_{1}-w_{2}\right) \mathrm{d} x \leqslant c \varepsilon .
$$

We conclude as in [5]. Letting $\varepsilon$ tend to zero, we obtain

$$
\int_{\Omega \cap\left\{w_{1}>w_{2}\right\}} w_{1} w_{2}\left(\frac{f\left(w_{2}\right)}{w_{2}}-\frac{f\left(w_{1}\right)}{w_{1}}\right) \mathrm{d} x \leqslant 0,
$$

which, together with the hypothesis on $f$, gives $w_{1} \leqslant w_{2}$ in $\Omega$. Comparison in $C_{\Omega}$ follows easily by the maximum principle.

Now we show that the solutions to problem (5.1)-(5.4) are bounded and Hölder continuous. In $\S 5.4$ we obtain a uniform $L^{\infty}$-estimate in the case where $f$ is given by (5.3) and the convex power is subcritical.

Proposition 5.4. Let $f$ satisfy (5.4) with $p<(N+\alpha) /(N-\alpha)$, and let $w \in$ $X_{0}^{\alpha}\left(C_{\Omega}\right)$ be an energy solution to problem (5.1). Then $w \in L^{\infty}\left(C_{\Omega}\right) \cap \mathcal{C}^{\gamma}\left(\bar{C}_{\Omega}\right)$ for some $0<\gamma<1$.

Proof. The proof closely follows the technique of [12]. As in the proof of theorem 4.7, we assume $w \geqslant 0$. We consider, formally, the test function $\varphi=w^{\beta-p}$, for some $\beta>p+1$. The justification of the following calculations can be made substituting $\varphi$ by some approximated truncation. We therefore proceed with the formal analysis. We obtain, using the trace immersion, the inequality

$$
\left(\int_{\Omega} w^{(\beta-p+1) N /(N-\alpha)}\right)^{(N-\alpha) / N} \leqslant C(\beta, \alpha, N, \Omega) \int_{\Omega} w^{\beta} .
$$

This estimate allows us to obtain the following iterative process

$$
\|w\|_{\beta_{j+1}} \leqslant C\|w\|_{\beta_{j}}^{\beta_{j} /\left(\beta_{j}-p+1\right)}
$$

with $\beta_{j+1}=N /(N-\alpha)\left(\beta_{j}+1-p\right)$. To have $\beta_{j+1}>\beta_{j}$ we need $\beta_{j}>(p-1) N /(\alpha)$. Since $w \in L^{2_{\alpha}^{*}}(\Omega)$, starting with $\beta_{0}=2 N /(N-\alpha)$, we obtain the above restriction provided $p<(N+\alpha) /(N-\alpha)$. It is clear that, in a finite number of steps, we get, for $g(x)=f(w(x, 0))$, the regularity $g \in L^{r}$ for some $r>N / \alpha$. As a consequence, we obtain the conclusion by applying theorem 4.7 and corollary 4.8 .

\subsection{A non-existence result}

The following result relies on the use of a classical Pohozaev-type multiplier. 
Proposition 5.5. Assume that $f$ is a continuous function with primitive $F$, and $w$ is a bounded energy solution to problem (5.1). Then the following Pohozaev-type identity holds

$$
\frac{1}{2} \int_{\partial_{\mathrm{L}} C_{\Omega}} y^{1-\alpha}\langle x, \nu\rangle|\nabla w|^{2} \mathrm{~d} \sigma-N \int_{\Omega} F(w) \mathrm{d} x+\frac{1}{2}(N-\alpha) \int_{\Omega} w f(w) \mathrm{d} x=0,
$$

where $\nu$ is the (exterior) normal vector to $\partial \Omega$.

Proof. Just use the identity

$$
\begin{aligned}
\langle x, \nu\rangle y^{\alpha-1} \operatorname{div}\left(y^{1-\alpha} \nabla w\right)+\operatorname{div}\left[y^{1-\alpha}(\langle(x, y), \nabla w\rangle\right. & \left.\left.\nabla w-\frac{1}{2}(x, y)|\nabla w|^{2}\right)\right] \\
& +\left(\frac{1}{2}(N+2-\alpha)-1\right)|\nabla w|^{2}=0 .
\end{aligned}
$$

This equality for $\mathcal{C}^{2}$ functions can be checked using calculus. For energy solutions we use a classical approximation procedure.

As a consequence, we obtain a non-existence result in the supercritical case for domains with particular geometry.

THEOREM 5.6. If $\Omega$ is star-shaped and the nonlinearity $f, F$ are as in the previous proposition and satisfy the inequality $((N-\alpha) s f(s)-2 N F(s)) \geqslant 0$, then problem (5.1) has no bounded solution. In particular, in the case $f(s)=|s|^{p-1} s$, this means that there is no bounded solution for any $p \geqslant(N+\alpha) /(N-\alpha)$.

The case $\alpha=1$ has been proved in [15]. The corresponding result for the Laplacian (problem (1.1) with $\alpha=2$ ) comes from [38].

\subsection{Proof of theorem 1.1}

Here we prove theorem 1.1 in terms of the solution of the local version (5.1). For the sake of readability, we split the proof into several lemmas. From now on, we define

$$
\left.\begin{array}{rlrl}
-\operatorname{div}\left(y^{1-\alpha} \nabla w\right) & =0 & & \text { in } C_{\Omega}, \\
w & =0 & & \text { on } \partial_{\mathrm{L}} C_{\Omega}, \\
\frac{\partial w}{\partial \nu^{\alpha}} & =\lambda w^{q}+w^{p}, & & w>0 \text { in } \Omega,
\end{array}\right\}
$$

and consider the associated energy functional

$$
J_{\lambda}(w)=\frac{1}{2} \int_{C_{\Omega}} y^{1-\alpha}|\nabla w|^{2} \mathrm{~d} x \mathrm{~d} y-\int_{\Omega} F_{\lambda}(w) \mathrm{d} x
$$

where

$$
F_{\lambda}(s)=\frac{\lambda}{q+1} s^{q+1}+\frac{1}{p+1} s^{p+1} .
$$

Lemma 5.7. Let $\Lambda$ be defined by

$$
\Lambda=\sup \left\{\lambda>0: \text { problem }\left(P_{\lambda}\right) \text { has a solution }\right\} .
$$

Then $0<\Lambda<\infty$. 
Proof. Consider the eigenvalue problem associated to the first eigenvalue $\lambda_{1}$, and let $\varphi_{1}>0$ be an associated eigenfunction (see lemma 4.3). Then, using $\varphi_{1}$ as a test function in $\left(P_{\lambda}\right)$, we have that

$$
\int_{\Omega}\left(\lambda w^{q}+w^{p}\right) \varphi_{1} \mathrm{~d} x=\lambda_{1} \int_{\Omega} w \varphi_{1} \mathrm{~d} x
$$

Since there exist positive constants $c, \delta$ such that $\lambda t^{q}+t^{p}>c \lambda^{\delta} t$, for any $t>0$ we obtain from (5.5) (recall that $w>0$ ) that $c \lambda^{\delta}<\lambda_{1}$, which implies $\Lambda<\infty$.

To prove $\Lambda>0$, we use the sub- and supersolution technique to construct a solution for any small $\lambda$. In fact, a subsolution is obtained as $\underline{w}=\varepsilon \varphi_{1}, \varepsilon>0$ small. A supersolution is a suitable multiple of the function $g$ solution to

$$
\begin{aligned}
-\operatorname{div}\left(y^{1-\alpha} \nabla g\right) & =0 & & \text { in } C_{\Omega}, \\
g & =0 & & \text { on } \partial_{\mathrm{L}} C_{\Omega}, \\
\frac{\partial g}{\partial \nu^{\alpha}} & =1 & & \text { in } \Omega .
\end{aligned}
$$

This proves the third statement in theorem 1.1.

Lemma 5.8. Problem $\left(P_{\lambda}\right)$ has at least a positive solution for every $0<\lambda<\Lambda$. Moreover, the family $\left\{w_{\lambda}\right\}$ of minimal solutions is increasing with respect to $\lambda$.

REMARK 5.9. Although this $\Lambda$ is not exactly the same as that of theorem 1.1 (see remark 5.1), we have not changed the notation for the sake of simplicity.

Proof of lemma 5.8. We already proved in the previous lemma that problem $\left(P_{\lambda}\right)$ has a solution for every small $\lambda>0$. Another way of proving this result is to look at the associated functional $J_{\lambda}$. Using theorem 4.4, we have that this functional verifies

$$
\begin{aligned}
J_{\lambda}(w)= & \frac{1}{2} \int_{C_{\Omega}} y^{1-\alpha}|\nabla w|^{2} \mathrm{~d} x \mathrm{~d} y-\int_{\Omega} F_{\lambda}(w) \mathrm{d} x \\
\geqslant & \frac{1}{2} \int_{C_{\Omega}} y^{1-\alpha}|\nabla w|^{2} \mathrm{~d} x \mathrm{~d} y-\lambda C_{1}\left(\int_{C_{\Omega}} y^{1-\alpha}|\nabla w|^{2} \mathrm{~d} x \mathrm{~d} y\right)^{(q+1) / 2} \\
& \quad-C_{2}\left(\int_{C_{\Omega}} y^{1-\alpha}|\nabla w|^{2} \mathrm{~d} x \mathrm{~d} y\right)^{(p+1) / 2},
\end{aligned}
$$

for some positive constants $C_{1}$ and $C_{2}$. Then, for sufficiently small $\lambda$, there exist two solutions of problem $\left(P_{\lambda}\right)$, one given by minimization and another given by the mountain-pass theorem [4]. The proof is standard, based on the geometry of the function $g(t)=\frac{1}{2} t^{2}-\lambda C_{1} t^{q+1}-C_{2} t^{p+1}$ (see, for example, [27] for more details). This in particular proves $\Lambda>0$.

We now show that there exists a solution for every $\lambda \in(0, \Lambda)$. Later in the paper (see lemma 5.11), we shall prove that there are in fact at least two solutions in the whole interval $(0, \Lambda)$.

By definition of $\Lambda$, we know that there exists a solution corresponding to any value of $\lambda$ close to $\Lambda$. Let us denote it by $\mu$, and let $w_{\mu}$ be the associated solution. Now $w_{\mu}$ is a supersolution for all problems $\left(P_{\lambda}\right)$ with $\lambda<\mu$. Take $v_{\lambda}$ as the unique solution 
to problem (5.1) with $f(s)=\lambda s^{q}$. Obviously, $v_{\lambda}$ is a subsolution to problem $\left(P_{\lambda}\right)$. By lemma 5.3, $v_{\lambda} \leqslant w_{\mu}$. Therefore, by lemma 5.2, we conclude that there is a solution for all $\lambda \in(0, \mu)$ and, as a consequence, for the whole open interval $(0, \Lambda)$. Moreover, this solution is the minimal one. The monotonicity follows directly from the comparison lemma.

This proves the first statement in theorem 1.1.

Lemma 5.10. Problem $\left(P_{\lambda}\right)$ has at least one solution if $\lambda=\Lambda$.

Proof. Let $\left\{\lambda_{n}\right\}$ be a sequence such that $\lambda_{n} \nearrow \Lambda$. We denote by $w_{n}=w_{\lambda_{n}}$ the minimal solution to problem $\left(P_{\lambda_{n}}\right)$. As in [5], we can prove that the linearized equation at the minimal solution has non-negative eigenvalues. Then it follows, as in [5], that $J_{\lambda_{n}}\left(w_{n}\right)<0$. Since $J_{\lambda_{n}}^{\prime}\left(w_{n}\right)=0$, we easily obtain the bound $\left\|w_{n}\right\|_{X_{0}^{\alpha}\left(C_{\Omega}\right)} \leqslant k$. Hence, there exists a weakly convergent subsequence in $X_{0}^{\alpha}\left(C_{\Omega}\right)$ and, as a consequence, a weak solution of $\left(P_{\lambda}\right)$ for $\lambda=\Lambda$.

This proves the second statement in theorem 1.1.

To conclude the proof of theorem 1.1, next we show the existence of a second solution for every $0<\lambda<\Lambda$. It is essential to have that the first solution is given as a local minimum of the associated functional, $J_{\lambda}$. To prove this last assertion we follow some ideas developed in [2].

Lemma 5.11. Problem $\left(P_{\lambda}\right)$ has at least two solutions for each $\lambda \in(0, \Lambda)$.

Proof. Let $\lambda_{0} \in(0, \Lambda)$ be fixed and consider $\lambda_{0}<\bar{\lambda}_{1}<\Lambda$. Take $\phi_{0}=w_{\lambda_{0}}, \phi_{1}=w_{\bar{\lambda}_{1}}$ as the two minimal solutions to problem $\left(P_{\lambda}\right)$ with $\lambda=\lambda_{0}$ and $\lambda=\bar{\lambda}_{1}$, respectively, then, by comparison, $\phi_{0}<\phi_{1}$. We define

$$
M=\left\{w \in X_{0}^{\alpha}\left(C_{\Omega}\right): 0 \leqslant w \leqslant \phi_{1}\right\} .
$$

Note that $M$ is a convex closed set of $X_{0}^{\alpha}\left(C_{\Omega}\right)$. Since $J_{\lambda_{0}}$ is bounded from below in $M$ and it is semicontinuous on $M$, we obtain the existence of $\underline{\omega} \in M$ such that $J_{\lambda_{0}}(\underline{\omega})=\inf _{w \in M} J_{\lambda_{0}}(w)$. Let $v_{0}$ be the unique positive solution to problem

$$
\left.\begin{array}{rlrl}
-\operatorname{div}\left(y^{1-\alpha} \nabla v_{0}\right) & =0 & & \text { in } C_{\Omega}, \\
v_{0} & =0 & & \text { on } \partial_{\mathrm{L}} C_{\Omega}, \\
\frac{\partial v_{0}}{\partial \nu^{\alpha}} & =v_{0}^{q} & & \text { in } \Omega .
\end{array}\right\}
$$

The existence and uniqueness of this solution is clear (see lemma 5.3). Since, for $0<\varepsilon \ll \lambda_{0}$ and $J_{\lambda_{0}}\left(\varepsilon v_{0}\right)<0$, we have $\varepsilon v_{0} \in M$, then $\underline{\omega} \neq 0$. Therefore, $J_{\lambda_{0}}(\underline{\omega})<0$. Using arguments similar to those in [42, theorem 2.4], we obtain that $\underline{\omega}$ is a solution to problem $\left(P_{\lambda_{0}}\right)$. There are two possibilities.

- If $\underline{\omega} \not \equiv w_{\lambda_{0}}$, then the result follows.

- If $\underline{\omega} \equiv w_{\lambda_{0}}$, we just have to prove that $\underline{\omega}$ is a local minimum of $J_{\lambda_{0}}$. Assuming that this is true, the conclusion in part (iv) of theorem 1.1 follows by using a classical argument. The second solution is given by the mountain-pass theorem (see, for example, [4]). 
We now prove that the minimal solution $w_{\lambda_{0}}$ is in fact a local minimum of $J_{\lambda_{0}}$. We argue by contradiction.

Suppose that $\underline{\omega}$ is not a local minimum of $J_{\lambda_{0}}$ in $X_{0}^{\alpha}\left(C_{\Omega}\right)$. Then there exists a sequence $\left\{v_{n}\right\} \subset X_{0}^{\alpha}\left(C_{\Omega}\right)$ such that $\left\|v_{n}-\underline{\omega}\right\|_{X_{0}^{\alpha}} \rightarrow 0$ and $J_{\lambda_{0}}\left(v_{n}\right)<J_{\lambda_{0}}(\underline{\omega})$.

Let $w_{n}=\left(v_{n}-\phi_{1}\right)^{+}$and $z_{n}=\max \left\{0, \min \left\{v_{n}, \phi_{1}\right\}\right\}$. It is clear that $z_{n} \in M$ and

$$
z_{n}(x, y)= \begin{cases}0 & \text { if } v_{n}(x, y) \leqslant 0 \\ v_{n}(x, y) & \text { if } 0 \leqslant v_{n}(x, y) \leqslant \phi_{1}(x, y), \\ \phi_{1}(x, y) & \text { if } \phi_{1}(x, y) \leqslant v_{n}(x, y) .\end{cases}
$$

We set

$$
\begin{array}{ll}
T_{n} \equiv\left\{(x, y) \in C_{\Omega}: z_{n}(x, y)=v_{n}(x, y)\right\}, & S_{n} \equiv \operatorname{supp}\left(w_{n}\right), \\
\tilde{T}_{n}=\bar{T}_{n} \cap \Omega, & \tilde{S}_{n}=S_{n} \cap \Omega .
\end{array}
$$

Note that $\operatorname{supp}\left(v_{n}^{+}\right)=T_{n} \cup S_{n}$. We claim that

$$
\left|\tilde{S}_{n}\right|_{\Omega} \rightarrow 0 \text { as } n \rightarrow \infty,
$$

where

$$
|A|_{\Omega} \equiv \int_{\Omega} \chi_{A}(x) \mathrm{d} x .
$$

By the definition of $F_{\lambda}$, we set

$$
F_{\lambda_{0}}(s)=\frac{l_{0}}{q+1} s_{+}^{q+1}+\frac{1}{p+1} s_{+}^{p+1} \quad \text { for } s \in \mathbb{R},
$$

and obtain

$$
\begin{aligned}
J_{\lambda_{0}}\left(v_{n}\right)= & \frac{1}{2} \int_{C_{\Omega}} y^{1-\alpha}\left|\nabla v_{n}\right|^{2} \mathrm{~d} x \mathrm{~d} y-\int_{\Omega} F_{\lambda_{0}}\left(v_{n}\right) \mathrm{d} x \\
= & \frac{1}{2} \int_{T_{n}} y^{1-\alpha}\left|\nabla z_{n}\right|^{2} \mathrm{~d} x \mathrm{~d} y-\int_{\tilde{T}_{n}} F_{\lambda_{0}}\left(z_{n}\right) \mathrm{d} x+\frac{1}{2} \int_{S_{n}} y^{1-\alpha}\left|\nabla v_{n}\right|^{2} \mathrm{~d} x \mathrm{~d} y \\
& -\int_{\tilde{S}_{n}} F_{\lambda_{0}}\left(v_{n}\right) \mathrm{d} x+\frac{1}{2} \int_{C_{\Omega}} y^{1-\alpha}\left|\nabla v_{n}^{-}\right|^{2} \mathrm{~d} x \mathrm{~d} y \\
= & \frac{1}{2} \int_{T_{n}} y^{1-\alpha}\left|\nabla z_{n}\right|^{2} \mathrm{~d} x \mathrm{~d} y-\int_{\tilde{T}_{n}} F_{\lambda_{0}}\left(z_{n}\right) \mathrm{d} x \\
& +\frac{1}{2} \int_{S_{n}} y^{1-\alpha}\left|\nabla\left(w_{n}+\phi_{1}\right)\right|^{2} \mathrm{~d} x \mathrm{~d} y-\int_{\tilde{S}_{n}} F_{\lambda_{0}}\left(w_{n}+\phi_{1}\right) \mathrm{d} x \\
& +\frac{1}{2} \int_{C_{\Omega}} y^{1-\alpha}\left|\nabla v_{n}^{-}\right|^{2} \mathrm{~d} x \mathrm{~d} y .
\end{aligned}
$$

Since

$$
\int_{C_{\Omega}} y^{1-\alpha}\left|\nabla z_{n}\right|^{2} \mathrm{~d} x \mathrm{~d} y=\int_{T_{n}} y^{1-\alpha}\left|\nabla v_{n}\right|^{2} \mathrm{~d} x \mathrm{~d} y+\int_{S_{n}} y^{1-\alpha}\left|\nabla \phi_{1}\right|^{2} \mathrm{~d} x \mathrm{~d} y
$$

and

$$
\int_{\Omega} F_{\lambda_{0}}\left(z_{n}\right) \mathrm{d} x=\int_{\tilde{T}_{n}} F_{\lambda_{0}}\left(v_{n}\right) \mathrm{d} x+\int_{\tilde{S}_{n}} F_{\lambda_{0}}\left(\phi_{1}\right) \mathrm{d} x
$$


by using the fact that $\phi_{1}$ is a supersolution to $\left(P_{\lambda}\right)$ with $l=\lambda_{0}$, we conclude that

$$
\begin{aligned}
J_{\lambda_{0}}\left(v_{n}\right)= & J_{\lambda_{0}}\left(z_{n}\right)+\frac{1}{2} \int_{S_{n}} y^{1-\alpha}\left(\left|\nabla\left(w_{n}+\phi_{1}\right)\right|^{2}-\left|\nabla \phi_{1}\right|^{2}\right) \mathrm{d} x \mathrm{~d} y \\
& \quad-\int_{\tilde{S}_{n}}\left(F_{\lambda_{0}}\left(w_{n}+\phi_{1}\right)-F_{\lambda_{0}}\left(\phi_{1}\right)\right) \mathrm{d} x+\frac{1}{2} \int_{C_{\Omega}} y^{1-\alpha}\left|\nabla v_{n}^{-}\right|^{2} \mathrm{~d} x \mathrm{~d} y \\
\geqslant & J_{\lambda_{0}}\left(z_{n}\right)+\frac{1}{2}\left\|w_{n}\right\|_{X_{0}^{\alpha}}^{2}+\frac{1}{2}\left\|v_{n}^{-}\right\|_{X_{0}^{\alpha}}^{2} \\
& \quad-\int_{\Omega}\left\{F_{\lambda_{0}}\left(w_{n}+\phi_{1}\right)-F_{\lambda_{0}}\left(\phi_{1}\right)-\left(F_{\lambda_{0}}\right)_{u}\left(\phi_{1}\right) w_{n}\right\} \mathrm{d} x \\
\geqslant & J_{\lambda_{0}}(\underline{\omega})+\frac{1}{2}\left\|w_{n}\right\|_{X_{0}^{\alpha}}^{2}+\frac{1}{2}\left\|v_{n}^{-}\right\|_{X_{0}^{\alpha}}^{2} \\
& \quad-\int_{\Omega}\left\{F_{\lambda_{0}}\left(w_{n}+\phi_{1}\right)-F_{\lambda_{0}}\left(\phi_{1}\right)-\left(F_{\lambda_{0}}\right)_{u}\left(\phi_{1}\right) w_{n}\right\} \mathrm{d} x .
\end{aligned}
$$

On one hand, taking into account that $0<q+1<2$, we obtain that

$$
0 \leqslant \frac{1}{q+1}\left(w_{n}+\phi_{1}\right)^{q+1}-\frac{1}{q+1} \phi_{1}^{q+1}-\phi_{1}^{q} w_{n} \leqslant \frac{1}{2} q \frac{w_{n}^{2}}{\phi_{1}^{1-q}} .
$$

The well-known Picone inequality [37] establishes

$$
|\nabla u|^{2}-\nabla\left(\frac{u^{2}}{v}\right) \cdot \nabla v \geqslant 0
$$

for differentiable functions $v>0, u \geqslant 0$. In our case, by an approximation argument, we obtain

$$
\lambda_{0} \int_{\Omega} \frac{w_{n}^{2}}{\phi_{1}^{1-q}} \mathrm{~d} x \leqslant\left\|w_{n}\right\|_{X_{0}^{\alpha}}^{2} .
$$

On the other hand, since $p+1>2$,

$$
\begin{aligned}
0 & \leqslant \frac{1}{p+1}\left(w_{n}+\phi_{1}\right)^{p+1}-\frac{1}{p+1} \phi_{1}^{p+1}-\phi_{1}^{p} w_{n} \\
& \leqslant \frac{1}{2} r w_{n}^{2}\left(w_{n}+\phi_{1}\right)^{p-1} \\
& \leqslant C(p)\left(\phi_{1}^{p-1} w_{n}^{2}+w_{n}^{p+1}\right) .
\end{aligned}
$$

Hence, using that $p+1<2_{\alpha}^{*}$ and claim (5.7),

$$
\int_{\Omega}\left\{\frac{1}{p+1}\left(w_{n}+\phi_{1}\right)^{p+1}-\frac{1}{p+1} \phi_{1}^{p+1}-\phi_{1}^{p} w_{n}\right\} \mathrm{d} x \leqslant o(1)\left\|w_{n}\right\|_{X_{0}^{\alpha}}^{2} .
$$

As a consequence, we obtain that

$$
\begin{aligned}
J_{\lambda_{0}}\left(v_{n}\right) & \geqslant J_{\lambda_{0}}(\underline{\omega})+\frac{1}{2}\left\|w_{n}\right\|_{X_{0}^{\alpha}}^{2}(1-q-o(1))+\frac{1}{2}\left\|v_{n}^{-}\right\|_{X_{0}^{\alpha}}^{2} \\
& \equiv J_{\lambda_{0}}(\underline{\omega})+\frac{1}{2}\left\|w_{n}\right\|_{X_{0}^{\alpha}}^{2}(1-q-o(1))+o(1) .
\end{aligned}
$$

Since $q<1$, it results that

$$
J_{\lambda_{0}}(\underline{\omega})>J_{\lambda_{0}}\left(v_{n}\right) \geqslant J_{\lambda_{0}}(\underline{\omega}) \text { for } n>n_{0},
$$

which is a contradiction with the main hypothesis. Hence, $\underline{\omega}$ is a minimum. 
To complete the proof we have to prove claim (5.7). For small $\varepsilon>0$, and for $\delta>0$ ( $\delta$ to be chosen later $)$, we consider

$$
\begin{aligned}
& E_{n}=\left\{x \in \Omega: v_{n}(x) \geqslant \phi_{1}(x) \wedge \phi_{1}(x)>\underline{\omega}(x)+\delta\right\}, \\
& F_{n}=\left\{x \in \Omega: v_{n}(x) \geqslant \phi_{1}(x) \wedge \phi_{1}(x) \leqslant \underline{\omega}(x)+\delta\right\} .
\end{aligned}
$$

Using the fact that

$$
\begin{aligned}
0 & =\left|\left\{x \in \Omega: \phi_{1}(x)<\underline{\omega}(x)\right\}\right| \\
& =\left|\bigcap_{j=1}^{\infty}\left\{x \in \Omega: \phi_{1}(x) \leqslant \underline{\omega}(x)+\frac{1}{j}\right\}\right| \\
& =\lim _{j \rightarrow \infty}\left|\left\{x \in \Omega: \phi_{1}(x) \leqslant \underline{\omega}(x)+\frac{1}{j}\right\}\right|,
\end{aligned}
$$

we obtain, for sufficiently large $j_{0}$, that if $\delta<1 / j_{0}$, then

$$
\left|\left\{x \in \Omega: \phi_{1}(x) \leqslant \underline{\omega}(x)+\delta\right\}\right| \leqslant \frac{1}{2} \varepsilon .
$$

Hence, we conclude that $\left|F_{n}\right|_{\Omega} \leqslant \frac{1}{2} \varepsilon$.

Since $\left\|v_{n}-\underline{\omega}\right\|_{X_{0}^{\alpha}} \rightarrow 0$ as $n \rightarrow \infty$, particularly by the trace embedding

$$
\left\|v_{n}-\underline{\omega}\right\|_{L^{2}(\Omega)} \rightarrow 0
$$

we obtain that, for large $n \geqslant n_{0}$,

$$
\frac{1}{2} \delta^{2} \varepsilon \geqslant \int_{C_{\Omega}}\left|v_{n}-\underline{\omega}\right|^{2} \mathrm{~d} x \geqslant \int_{E_{n}}\left|v_{n}-\underline{\omega}\right|^{2} \mathrm{~d} x \geqslant \delta^{2}\left|E_{n}\right|_{\Omega} .
$$

Therefore, $\left|E_{n}\right|_{\Omega} \leqslant \frac{1}{2} \varepsilon$. Since $\tilde{S}_{n} \subset F_{n} \cup E_{n}$, we conclude that $\left|\tilde{S}_{n}\right|_{\Omega} \leqslant \varepsilon$ for $n \leqslant n_{0}$. Hence, $\left|\tilde{S}_{n}\right|_{\Omega} \rightarrow 0$ as $n \rightarrow \infty$, and the claim follows.

\subsection{Proof of theorem 1.2 and further results}

We start with the uniform $L^{\infty}$-estimates for solutions to problem (1.1) in its local version given by $\left(P_{\lambda}\right)$.

Theorem 5.12. Assume $\alpha \geqslant 1, p<(N+\alpha) /(N-\alpha)$ and $N \geqslant 2$. Then there exists a constant $C=C(p, \Omega)>0$ such that every solution to problem $\left(P_{\lambda}\right)$ satisfies

$$
\|w\|_{\infty} \leqslant C
$$

for every $0 \leqslant \lambda \leqslant \Lambda$.

The proof is based on a scaling method of [28] and two non-existence results (see theorems 3.1 and 3.4).

Proof of theorem 5.12. By contradiction, assume that there exists a sequence $\left\{w_{n}\right\} \subset X_{0}^{\alpha}\left(C_{\Omega}\right)$ of solutions to $\left(P_{\lambda}\right)$ verifying that $M_{n}=\left\|w_{n}\right\|_{\infty} \rightarrow \infty$ as $n \rightarrow \infty$. By the maximum principle, which holds for our problem [26], the maximum of $w_{n}$ is attained at a point $\left(x_{n}, 0\right)$, where $x_{n} \in \Omega$. We define $\Omega_{n}=1 / \mu_{n}\left(\Omega-x_{n}\right)$, with $\mu_{n}=M_{n}^{(1-p) / \alpha}$, i.e. we centre at $x_{n}$ and dilate by $1 / \mu_{n} \rightarrow \infty$ as $n \rightarrow \infty$. 
We consider the scaled functions

$$
v_{n}(x, y)=\frac{w_{n}\left(x_{n}+\mu_{n} x, \mu_{n} y\right)}{M_{n}} \text { for } x \in \Omega_{n}, y \geqslant 0 .
$$

It is clear that $\left\|v_{n}\right\| \leqslant 1, v_{n}(0,0)=1$ and, moreover,

$$
\left.\begin{array}{rlrl}
-\operatorname{div}\left(y^{1-\alpha} \nabla v_{n}\right) & =0 & & \text { in } C_{\Omega_{n}}, \\
v_{n} & =0 & & \text { on } \partial_{\mathrm{L}} C_{\Omega_{n}}, \\
\frac{\partial v_{n}}{\partial \nu^{\alpha}} & =\lambda M_{n}^{q-p} v_{n}^{q}+v_{n}^{p} & & \text { in } \Omega_{n} \times\{0\} .
\end{array}\right\}
$$

By the Arzelà-Ascoli theorem (the solution is $\mathcal{C}^{\gamma}$; see proposition 5.4), there exists a subsequence, which we again denote by $v_{n}$, which converges to some function $v$ as $n \rightarrow \infty$. In order to see the problem satisfied by $v$ we pass to the limit in the weak formulation of (5.8). We observe that $\left\|v_{n}\right\|_{\infty} \leqslant 1$ implies $\left\|v_{n}\right\|_{X_{0}^{\alpha}\left(C_{\Omega}\right)} \leqslant C$, since

$$
\int_{C_{\Omega}} y^{1-\alpha}\left|\nabla v_{n}\right|^{2}=\lambda M_{n}^{q-p} \int_{\Omega} v_{n}^{q+1}+\int_{\Omega} v_{n}^{p+1} \leqslant C .
$$

Defining $d_{n}=\operatorname{dist}\left(x_{n}, \partial \Omega\right)$, there are two possibilities as $n \rightarrow \infty$ with regard to the behaviour of the ratio $d_{n} / \mu_{n}$ :

(i) $\left\{d_{n} / \mu_{n}\right\}_{n}$ is not bounded;

(ii) $\left\{d_{n} / \mu_{n}\right\}_{n}$ remains bounded.

In the first case, since $B_{d_{n} / \mu_{n}}(0) \subset \Omega_{n}$, and $\Omega_{n}$ is smooth, it is clear that $\Omega_{n}$ tends to $\mathbb{R}^{N}$ and $v$ is a solution to

$$
\begin{aligned}
-\operatorname{div}\left(y^{1-\alpha} \nabla v\right) & =0 & & \text { in } \mathbb{R}_{+}^{N+1}, \\
\frac{\partial v}{\partial \nu^{\alpha}} & =v^{p} & & \text { on } \partial \mathbb{R}_{+}^{N+1} .
\end{aligned}
$$

Moreover, $v(0,0)=1$ and $v>0$, which is a contradiction with theorem 3.1.

In the second case, we may assume that $d_{n} / \mu_{n} \rightarrow s \geqslant 0$ as $n \rightarrow \infty$. As a consequence, passing to the limit, the domains $\Omega_{n}$ converge (up to a rotation) to some half-space $H_{s}=\left\{x \in \mathbb{R}^{N}: x_{N}>-s\right\}$. Here we obtain that $v$ is a solution to

$$
\begin{aligned}
-\operatorname{div}\left(y^{1-\alpha} \nabla v\right) & =0 & & \text { in } H_{s} \times(0, \infty), \\
\frac{\partial v}{\partial \nu^{\alpha}} & =v^{p} & & \text { on } H_{s} \times\{0\},
\end{aligned}
$$

with $\|v\|_{\infty}=1, v(0,0)=1$. In the case where $s=0$, this is a contradiction with the continuity of $v$. If $s>0$, the contradiction comes from theorem 3.4.

Next we prove a uniqueness result for solutions with a small norm.

THEOREM 5.13. There exists at most one solution to problem $\left(P_{\lambda}\right)$ with a small norm.

We follow the arguments in [5] closely, thereby establishing the following result. 
Lemma 5.14. Let $z$ be the unique solution to problem (5.6). There exists a constant $\beta>0$ such that

$$
\|\phi\|_{X_{0}^{\alpha}\left(C_{\Omega}\right)}^{2}-q \int_{\Omega} z^{q-1} \phi^{2} \mathrm{~d} x \geqslant \beta\|\phi\|_{L^{2}(\Omega)}^{2} \quad \text { for all } \phi \in X_{0}^{\alpha}\left(C_{\Omega}\right) .
$$

Proof. We recall that $z$ can be obtained by minimization as follows:

$$
\min \left\{\frac{1}{2}\|\omega\|_{X_{0}^{\alpha}\left(C_{\Omega}\right)}^{2}-\frac{1}{q+1}\|w\|_{L^{q+1}(\Omega)}^{q+1}: \omega \in X_{0}^{\alpha}\left(C_{\Omega}\right)\right\} .
$$

As a consequence,

$$
\|\phi\|_{X_{0}^{\alpha}\left(C_{\Omega}\right)}^{2}-q \int_{\Omega} z^{q-1} \phi^{2} \mathrm{~d} x \geqslant 0 \quad \text { for all } \phi \in X_{0}^{\alpha}\left(C_{\Omega}\right) .
$$

This implies that the first eigenvalue $a_{1}$ of the linearized problem

$$
\begin{aligned}
-\operatorname{div}\left(y^{1-\alpha} \nabla \phi\right) & =0 & & \text { in } C_{\Omega}, \\
\phi & =0 & & \text { on } \partial_{\mathrm{L}} C_{\Omega}, \\
\frac{\partial \phi}{\partial \nu^{\alpha}}-q z^{q-1} \phi & =a \phi & & \text { on } \Omega \times\{0\},
\end{aligned}
$$

is non-negative.

Assume first that $a_{1}=0$ and let $\varphi$ be a corresponding eigenfunction. Taking into account that $z$ is the solution to (5.6), we obtain that

$$
q \int_{\Omega} z^{q} \varphi \mathrm{d} x=\int_{\Omega} z^{q} \varphi \mathrm{d} x
$$

which is a contradiction.

Hence $a_{1}>0$, which proves (5.9).

Proof of theorem 5.13. Consider $A>0$ such that $p A^{p-1}<\beta$, where $\beta$ is given in (5.9). Now we prove that problem $\left(P_{\lambda}\right)$ has at most one solution with $L^{\infty}$-norm less than $A$.

Assume by contradiction that $\left(P_{\lambda}\right)$ has a second solution $w=w_{\lambda}+v$ verifying $\|w\|_{\infty}<A$. Since $w_{\lambda}$ is the minimal solution, it follows that $v>0$ in $\Omega \times[0, \infty)$. We now define $\eta=\lambda^{1 /(1-q)} z$, where $z$ is the solution to (5.6). Then it verifies $-\operatorname{div}\left(y^{1-\alpha} \nabla \eta\right)=0$ with boundary condition $\lambda \eta^{q}$. Moreover, $w_{\lambda}$ is a supersolution to the problem that $\eta$ verifies. Then, by comparison, lemma 5.3, applied with $f(t)=$ $\lambda t^{q}, v=\eta$ and $w=w_{\lambda}$, yields

$$
w_{\lambda} \geqslant \lambda^{1 /(1-q)} z \quad \text { on } \Omega \times\{0\} .
$$

Since $w=w_{\lambda}+v$ is a solution to $\left(P_{\lambda}\right)$, we have, on $\Omega \times\{0\}$,

$$
\begin{aligned}
\frac{\partial\left(w_{\lambda}+v\right)}{\partial \nu^{\alpha}} & =\lambda\left(w_{\lambda}+v\right)^{q}+\left(w_{\lambda}+v\right)^{p} \\
& \leqslant \lambda w_{\lambda}^{q}+\lambda q w_{\lambda}^{q-1} v+\left(w_{\lambda}+v\right)^{p}
\end{aligned}
$$


where the inequality is a consequence of the concavity; hence,

$$
\frac{\partial v}{\partial \nu^{\alpha}} \leqslant \lambda q w_{\lambda}^{q-1} v+\left(w_{\lambda}+v\right)^{p}-w_{\lambda}^{p} .
$$

Moreover, (5.10) implies $w_{\lambda}^{q-1} \geqslant \lambda^{-1} z^{q-1}$. From the previous two inequalities we obtain

$$
\frac{\partial v}{\partial \nu^{\alpha}} \leqslant q z^{q-1} v+\left(w_{\lambda}+v\right)^{p}-w_{\lambda}^{p} .
$$

Using that $\left\|w_{\lambda}+v\right\|_{\infty} \leqslant A$, we obtain $\left(w_{\lambda}+v\right)^{p}-w_{\lambda}^{p} \leqslant p A^{p-1} v$. As a consequence,

$$
\frac{\partial v}{\partial \nu^{\alpha}}-q z^{q-1} v \leqslant p A^{p-1} v
$$

Taking $v$ as a test function and $\phi=v$ in (5.9), we arrive at

$$
\beta \int_{\Omega} v^{2} \mathrm{~d} x \leqslant p A^{p-1} \int_{\Omega} v^{2} \mathrm{~d} x .
$$

Since $p A^{p-1}<\beta$, we conclude that $v \equiv 0$, which gives the desired contradiction.

REMARK 5.15. This proof also provides the asymptotic behaviour of $w_{\lambda}$ near $\lambda=0$, namely, $w_{\lambda} \approx \lambda^{1 /(1-q)} z$, where $z$ is the unique solution to problem (5.6).

\section{Acknowledgements}

C.B. and A.d.P. were partly supported by the MEC (Spain) Project MTM200806326-C02-02. E.C. was partly supported by the MEC (Spain) Project MTM200910878 .

\section{References}

1 B. Abdellaoui, E. Colorado and I. Peral. Effect of the boundary conditions in the behavior of the optimal constant of some Caffarelli-Kohn-Nirenberg inequalities: application to some doubly critical nonlinear elliptic problems. Adv. Diff. Eqns 11 (2006), 667-720.

2 S. Alama. Semilinear elliptic equations with sublinear indefinite nonlinearities. Adv. Diff. Eqns 4 (1999), 813-842.

3 A. Ambrosetti. Critical points and nonlinear variational problems. Mem. Soc. Math. France (N.S.) 49 (1992), 1-139.

4 A. Ambrosetti and P. H. Rabinowitz. Dual variational methods in critical point theory and applications. J. Funct. Analysis 14 (1973), 349-381.

5 A. Ambrosetti, H. Brézis and G. Cerami. Combined effects of concave and convex nonlinearities in some elliptic problems. J. Funct. Analysis 122 (1994), 519-543.

6 D. Applebaum. Lévy processes and stochastic calculus (2nd edn), Cambridge Studies in Advanced Mathematics, vol. 116 (Cambridge University Press, 2009).

7 B. Barrios, E. Colorado, A. de Pablo and U. Sánchez. On some critical problems for the fractional Laplacian operator. J. Diff. Eqns 252 (2012), 6133-6162.

8 J. Bertoin. Lévy processes, Cambridge Tracts in Mathematics, vol. 121 (Cambridge University Press, 1996).

9 L. Boccardo, M. Escobedo and I. Peral. A Dirichlet problem involving critical exponents. Nonlin. Analysis 24 (1995), 1639-1648.

10 K. Bogdan, K. Burdy and Z. Q. Chen. Censored stable processes. Prob. Theory Relat. Fields 127 (2003), 89-152.

11 H. Brézis and S. Kamin. Sublinear elliptic equation in $\mathbb{R}^{n}$. Manuscr. Math. 74 (1992), $87-106$. 
12 H. Brézis and T. Kato. Remarks on the Schrödinger operator with singular complex potentials. J. Math. Pures Appl. 9 (1979), 137-151.

13 H. Brézis and L. Nirenberg. Positive solutions of nonlinear elliptic equations involving critical Sobolev exponents. Commun. Pure Appl. Math. 36 (1983), 437-477.

14 X. Cabré and Y. Sire. Nonlinear equations for fractional Laplacians. I. Regularity, maximum principles and Hamiltonian estimates. Analysis PDE (In the press.)

15 X. Cabré and J. Tan. Positive solutions of nonlinear problems involving the square root of the Laplacian. Adv. Math. 224 (2010), 2052-2093.

16 L. A. Caffarelli and X. Cabré. Fully nonlinear elliptic equations, Colloquium Publications, vol. 43 (Providence, RI: American Mathematical Society, 1995).

17 L. A. Caffarelli and L. Silvestre. An extension problem related to the fractional Laplacian. Commun. PDEs 32 (2007), 1245-1260.

18 F. Charro, E. Colorado and I. Peral. Multiplicity of solutions to uniformly elliptic fully nonlinear equations with concave-convex right-hand side. J. Diff. Eqns 246 (2009), 42214248.

19 W. Chen, C. Li and B. Ou. Qualitative properties of solutions for an integral equation. Discrete Contin. Dynam. Syst. 12 (2005), 347-354.

20 W. Chen, C. Li and B. Ou. Classification of solutions for an integral equation. Commun. Pure Appl. Math. 59 (2006), 330-343.

21 M. Chipot, M. Chlebik, M. Fila and I. Shafir. Existence of positive solutions of a semilinear elliptic equation in $\mathbb{R}_{n}^{+}$with a nonlinear boundary condition. J. Math. Analysis Applic. 223 (1998), 429-471.

22 E. Colorado and I. Peral. Semilinear elliptic problems with mixed Dirichlet-Neumann boundary conditions. J. Funct. Analysis 199 (2003), 468-507.

23 R. Cont and P. Tankov. Financial modelling with jump processes (Boca Raton, FL: Chapman \& Hall/CRC, 2004).

24 A. Cotsiolis and N. K. Tavoularis. Best constants for Sobolev inequalities for higher order fractional derivatives. J. Math. Analysis Applic. 295 (2004), 225-236.

25 J. F. Escobar. Sharp constant in a Sobolev trace inequality. Indiana J. Math. 37 (1988), 687-698.

26 E. B. Fabes, C. E. Kenig and R. P. Serapioni. The local regularity of solutions of degenerate elliptic equations. Commun. PDEs 7 (1982), 77-116.

27 J. García Azorero and I. Peral. Multiplicity of solutions for elliptic problems with critical exponent or with non-symetric term. Trans. Am. Math. Soc. 323 (1991), 877-895.

28 B. Gidas and J. Spruck. A priori bounds for positive solutions of nonlinear elliptic equations. Commun. PDEs 6 (1981), 883-901.

29 B. Gidas, W. M. Ni and L. Nirenberg. Symmetry and related properties via the maximum principle. Commun. Math. Phys. 68 (1979), 209-243.

30 D. Gilbarg and N. S. Trudinger. Elliptic partial differential equations of second order, Classics in Mathematics Series (Springer, 2001).

31 Q. Y. Guan and Z. M. Ma. Reflected symmetric $\alpha$-stable processes and regional fractional Laplacian. Prob. Theory Relat. Fields 134 (2006), 649-694.

32 B. Hu. Nonexistence of a positive solution of the Laplace equation with a nonlinear boundary condition. Diff. Integ. Eqns 7 (1994), 301-313.

33 N. S. Landkof. Foundations of modern potential theory, Die Grundlehren der mathematischen Wissenschaften, vol. 180 (Springer, 1972).

34 N. N. Lebedev. Special functions and their applications (New York: Dover, 1972).

35 E. Lieb and M. Loss. Analysis (2nd edn), Graduate Studies in Mathematics, vol. 14 (Providence, RI: American Mathematical Society, 2001).

36 P.-L. Lions. The concentration-compactness principle in the calculus of variations. The limit case. II. Rev. Mat. Iber. 1 (1985), 45-121.

37 M. Picone. Sui valori eccezionali di un parametro da cui dipende un'equazione differenziale lineare ordinaria del second'ordine. Annali Scuola Norm. Sup. Pisa 11 (1910), 1-144.

38 S. Pohozahev. On the eigenfunctions of quasilinear elliptic problems. Mat. Sb. 82 (1970), 171-188.

39 J. Serrin. A symmetry problem in potential theory. Arch. Ration. Mech. Analysis 43 (1971), 304-318. 
40 E. M. Stein. Singular integrals and differentiability properties of functions, Princeton Mathematical Series, vol. 30 (Princeton University Press, 1970).

41 P. R. Stinga and J. L. Torrea. Extension problem and Harnack's inequality for some fractional operators. Commun. PDEs 35 (2010), 2092-2122.

42 M. Struwe. Variational methods: applications to nonlinear partial differential equations and Hamiltonian systems (Springer, 2000).

43 G. Talenti. Best constant in Sobolev inequality. Annali Mat. Pura Appl. 110 (1976), 353372.

44 L. Vlahos, H. Isliker, Y. Kominis and K. Hizonidis. Normal and anomalous diffusion: a tutorial, Order and Chaos (ed. T. Bountis), vol. 10 (Patras University Press, 2008).

45 J. Xiao. A sharp Sobolev trace inequality for the fractional-order derivatives. Bull. Sci. Math. 130 (2006), 87-96.

(Issued 15 February 2013) 